OPEN ACCESS

Edited by:

Daniel F. Gilbert,

Friedrich-Alexander-Universität Erlangen-Nürnberg, Germany

Reviewed by:

Timothy Lynagh,

University of Copenhagen, Denmark

Xiaojie Huang,

University of Pittsburgh, United States

${ }^{*}$ Correspondence:

Bert Brône

bert.brone@uhasselt.be

Svetlana M. Molchanova

svetlana.molchanova@helsinki.fi

${ }^{+}$Present address:

Giovanni Morelli,

Department of Neuroscience

and Brain Technologies, Istituto

Italiano di Tecnologia, Genoa, Italy

Claudia Bagni,

Department of Fundamental

Neurosciences, University of Lausanne, Lausanne, Switzerland

David Gall,

Laboratory of Physiology and Pharmacology, Université Libre de

Bruxelles, Brussels, Belgium

Svetlana M. Molchanova,

Molecular Neurology, Research Program Unit, University of Helsinki, Helsinki, Finland

\#These authors have contributed equally to this work

Received: 28 May 2018 Accepted: 24 September 2018

Published: 15 October 2018

Citation:

Comhair J, Devoght J, Morelli G, Harvey RJ, Briz V, Borrie SC, Bagni C,

Rigo J-M, Schiffmann SN, Gall D, Brône B and Molchanova SM (2018) Alpha2-Containing Glycine Receptors

Promote Neonatal Spontaneous Activity of Striatal Medium Spiny Neurons and Support Maturation of Glutamatergic Inputs.

Front. Mol. Neurosci. 11:380. doi: 10.3389/fnmol.2018.00380

\section{Alpha2-Containing Glycine Receptors Promote Neonatal Spontaneous Activity of Striatal Medium Spiny Neurons and Support Maturation of Glutamatergic Inputs}

\author{
Joris Comhair ${ }^{1,2}$, Jens Devoght ${ }^{2}$, Giovanni Morelli2t, Robert J. Harvey ${ }^{3,4}$, Victor Briz ${ }^{5,6}$, \\ Sarah C. Borrie ${ }^{5,6}$, Claudia Bagni ${ }^{5,6+}$, Jean-Michel Rigo², Serge N. Schiffmann', \\ David Gallit, Bert Brône ${ }^{2 * \neq}$ and Svetlana M. Molchanova ${ }^{1 * t \neq}$
}

1 Laboratory of Neurophysiology, ULB-Neuroscience Institute, Université Libre de Bruxelles, Brussels, Belgium, ${ }^{2}$ BIOMED Research Institute, University of Hasselt, Hasselt, Belgium, ${ }^{3}$ School of Health and Sport Sciences, University of the Sunshine Coast, Sippy Downs, QLD, Australia, ${ }^{4}$ Sunshine Coast Health Institute, Birtinya, QLD, Australia, ${ }^{5}$ Center for Human Genetics and Leuven Research Institute for Neuroscience and Disease, KU Leuven, Leuven, Belgium, ${ }^{6}$ VIB Center for the Biology of Disease, Leuven, Belgium

Glycine receptors (GlyRs) containing the a2 subunit are highly expressed in the developing brain, where they regulate neuronal migration and maturation, promote spontaneous network activity and subsequent development of synaptic connections. Mutations in GLRA2 are associated with autism spectrum disorder, but the underlying pathophysiology is not described yet. Here, using Glra2-knockout mice, we found a GlyR-dependent effect on neonatal spontaneous activity of dorsal striatum medium spiny neurons (MSNs) and maturation of the incoming glutamatergic innervation. Our data demonstrate that functional GlyRs are highly expressed in MSNs of one-week-old mice, but they do not generate endogenous chloride-mediated tonic or phasic current. Despite of that, knocking out the Glra2 severely affects the shape of action potentials and impairs spontaneous activity and the frequency of miniature AMPA receptormediated currents in MSNs. This reduction in spontaneous activity and glutamatergic signaling can attribute to the observed changes in neonatal behavioral phenotypes as seen in ultrasonic vocalizations and righting reflex. In adult Glra2-knockout animals, the glutamatergic synapses in MSNs remain functionally underdeveloped. The number of glutamatergic synapses and release probability at presynaptic site remain unaffected, but the amount of postsynaptic AMPA receptors is decreased. This deficit is a consequence of impaired development of the neuronal circuitry since acute inhibition of GlyRs by strychnine in adult MSNs does not affect the properties of glutamatergic synapses. Altogether, these results demonstrate that GlyR-mediated signaling supports neonatal spontaneous MSN activity and, in consequence, promotes the functional maturation of glutamatergic synapses on MSNs. The described mechanism might shed light on the pathophysiological mechanisms in GLRA2-linked autism spectrum disorder cases.

Keywords: autism spectrum disorders, dorsal striatum, medium spiny neurons, glycine receptors, spontaneous activity, synaptic development 


\section{INTRODUCTION}

Human subjects suffering from psychiatric disorders, including autism spectrum disorder (ASD) show defective connectivity in different brain regions and in many cases these disturbances in the neuronal network have a developmental origin (Yamasaki et al., 2017). Establishing the connectivity of neuronal networks is a process that is temporally and spatially regulated. For example, the formation and maturation of synaptic connections relies on recurring formation of neuronal protrusions (neurites and synapses). Furthermore, the activity of the neuron and local network in response to cell intrinsic programs and/or sensory experience are key regulators of synaptogenesis (Jamann et al., 2018). These findings have sparked an increased effort to characterize mechanisms that influence neuronal activity in the developing brain. Formation and refinement of glutamatergic synaptic connections occurs under the guidance of spontaneous electrical activity of new-born neurons (Cherubini et al., 2011; Huupponen et al., 2013). Activity-dependent maturation of synaptic connectivity has been described for many brain structures, including striatum (Dehorter et al., 2011; Kozorovitskiy et al., 2012). This activity propagates throughout the newly formed synaptic contacts, controlling post-translational modifications, promoting localized protein synthesis within dendrites and influencing gene transcription (Ebert and Greenberg, 2013). Mutations in genes that control these neuronal activity-dependent processes affect the maturation of synaptic connections (Lein et al., 2017).

Many neurotransmitter receptors, including glycine receptors (GlyRs), are known to be important regulators of neonatal spontaneous activity (Piton et al., 2011; Pilorge et al., 2016). GlyRs are ligand-gated chloride channels that are widely expressed throughout the brain and spinal cord. The diversity of the GlyRs results from a differential expression of four $\alpha$-subunits ( $\alpha 1-\alpha 4)$ and one $\beta$-subunit (Malosio et al., 1991) and the composition of the GlyRs differs between brain regions and developmental age (Avila et al., 2013a). GlyRs containing the $\alpha 2$ subunit are highly expressed in the developing brain, where they act as depolarizing neurotransmitter receptors (Kilb et al., 2002). They regulate migration and maturation of cortical neurons and promote neonatal spontaneous neuronal network activity, which is needed for the development of synaptic connections (Avila et al., 2013b, 2014). Several mutations in GLRA2 have been found in males with ASD, pointing at possible involvement of glycinergic transmission in development of cognitive abilities in these individuals (Pilorge et al., 2016). In a mouse model, deletion of Glra2 causes alterations in glutamatergic circuitry and synaptic plasticity in the cerebral cortex (Morelli et al., 2016).

We previously reported that GlyRs are expressed in adult striatal projection neurons (i.e., medium spiny neurons, MSNs) (Molchanova et al., 2018) that contain $\alpha 2$ as the main agonistbinding subunit and are tonically active at resting state. The main function of these GlyRs is to stabilize the resting membrane potential (RMP) and set the offset of action potential firing. In the present study, we aimed to evaluate the expression profile of GlyRs in the developing striatum and studied their effect on intrinsic cellular MSN parameters relating to the maturation and formation of glutamatergic synaptic connections.

\section{MATERIALS AND METHODS}

\section{Animals}

All animal experiments were conducted according to the ethical guidelines (in accordance with EU directive 2010/63/EU) provided by the University of Hasselt, the Universite Libre de Bruxelles and the KU Leuven. The reduction and refinement of the animals experiments was achieved by an ad hoc power calculation $(\mathrm{N})$ and designing paired experiments if possible. C57Bl/6 (both genders) and GlyR $\alpha 2$ subunit knockout mice (WTs and GlyRa2KOs, males) of specific ages were used in the study. GlyR $\alpha 2 \mathrm{KOs}$ were initially generated in the laboratory of Prof. Harvey and Prof. T. N. Dear by deletion of the exon 7 of Glra2. GlyR $\alpha 2 \mathrm{KOs}$ were fully back-crossed to C57Bl/6 background and genotyped as described previously (Avila et al., 2013a; D’Angelo et al., 1995).

\section{Immunohistochemistry, Imaging, and Analysis}

Neuronal identification and morphological analysis were performed by biocytin injection into cells after whole-cell patch-clamp recordings. Slices were fixed overnight in $4 \%$ PFA, after which they were washed with $0.01 \mathrm{M}$ phosphate-buffered saline (PBS in mM: $137 \mathrm{NaCl}, 2.7 \mathrm{KCl}, 10 \mathrm{NaH}_{2} \mathrm{PO}_{4}, 1.8$ $\mathrm{KH}_{2} \mathrm{PO}_{4}$ ) and permeabilized with $0.01 \mathrm{M}$ PBS $0.1 \%$ Triton-X 100 (Sigma-Aldrich) for $1.5 \mathrm{~h}$. Streptavidin Conjugate (1:500; A488; Life Technologies) was added to the permeabilization solution and incubated for $4 \mathrm{~h}$. Dendritic trees were imaged with a Plan-Apochromat $20 \times / 0.75$ objective, resolution of 2.3 pixels $/ \mu \mathrm{m}$ and Z-stack interval of $0.630 \mu \mathrm{m}$. Dendritic spines were imaged with a Plan-Apochromat $100 \times / 1.4$ oil DIC objective, resolution of 34.2 pixels $/ \mu \mathrm{m}$ and Z-stack interval of $0.258 \mu \mathrm{m}$. Sholl analysis was performed on 2D maximal intensity images acquired from Z-stacks, using the Fiji ImageJ plugin. The number of intersections was estimated with $10 \mu \mathrm{m}$ bins, and values per cell were used for statistical analysis. For spine density analysis, three distant dendrites were imaged per cell. Spine density was evaluated on the primary dendrites for P7 and on the secondary for P21 and adult animals. Values were plotted to a density per $20 \mu \mathrm{m}$ long section of the dendrite. Actual spine detection was done using the Fiji ImageJ plugin to quantify spines in a Z-stack image. Verification of the spine detection was done manually. The values for individual dendrites were averaged per cell. Values of spine density per cell were used for statistical analysis.

\section{Acute Brain Slice Preparation}

For electrophysiological recordings and morphology analysis, postnatal day (P7), P21 and adult (6 weeks to 3 months old) animals were used. Adult animals were deeply anesthetized using halothane before sacrifice by decapitation. Brains were rapidly removed and placed in ice-cold cutting solution of 
the following composition (in $\mathrm{mM}$ ): $140 \mathrm{C}_{5} \mathrm{H}_{14} \mathrm{ClNO}$ (choline chloride), $2.5 \mathrm{KCl}, 1.25 \mathrm{NaH}_{2} \mathrm{PO}_{4}, 7 \mathrm{MgCl}_{2}, 26 \mathrm{NaHCO}_{3}$, $0.5 \mathrm{CaCl}_{2}, 10 \mathrm{D}$-glucose equilibrated with $95 \% \mathrm{O}_{2}-5 \% \mathrm{CO}_{2}$ mixture. For recording NMDA mEPSCs, $\mathrm{MgCl}_{2}$ was excluded and $1.25 \mathrm{mM}$ of kynurenic acid was added. Depending on the experimental purpose, coronal, parasagittal, or horizontal (tilted at $30^{\circ}$ ) cortico-striatal slices were prepared on a vibratome (Leica VT1000S). Slice thickness varied depending on the age of the animals (300 $\mu \mathrm{m}$ for P7-P21, $220 \mu \mathrm{m}$ for adults). After preparation, slices were allowed to recover for $1 \mathrm{~h}$ at $34^{\circ} \mathrm{C}$ in artificial CSF with elevated $\mathrm{Mg}^{2+}$ concentration (unless NMDAmEPSCs were being recorded; composition in mM: $127 \mathrm{NaCl}$, $2.5 \mathrm{KCl}, 1.25 \mathrm{NaH}_{2} \mathrm{PO}_{4}, 3 \mathrm{MgCl}_{2}, 26 \mathrm{NaHCO}_{3}, 2 \mathrm{CaCl}_{2}, 15 \mathrm{D}$ glucose). All recordings were performed on MSNs located in the dorsal part of the striatum.

\section{Electrophysiology}

During recordings, slices were perfused with aCSF (composition in mM: $127 \mathrm{NaCl}, 2.5 \mathrm{KCl}, 1.25 \mathrm{NaH}_{2} \mathrm{PO}_{4}, 1 \mathrm{MgCl}_{2}, 26 \mathrm{NaHCO}_{3}$, $2 \mathrm{CaCl}_{2}, 15 \mathrm{D}$-glucose, $95 \% \mathrm{O}_{2}-5 \% \mathrm{CO}_{2}$ ) at a rate of $1-$ $2 \mathrm{ml} / \mathrm{min}$. To record NMDA-mEPSCs, $\mathrm{MgCl}_{2}$ was excluded and $25 \mu \mathrm{M}$ of $\mathrm{D}$-Serine together with $1 \mu \mathrm{M}$ of strychnine were added to aCSF. All recordings were made at $32^{\circ} \mathrm{C}$. Whole-cell patchclamp borosilicate-glass pipets (Hilgenberg) were obtained with a vertical two-stage puller (PIP 5; HEKA) and had a resistance typically between 5 and $7 \mathrm{M} \Omega$. Current- and voltage-clamp recordings were obtained with an EPC-10 amplifier (HEKA Elektronik, Germany) controlled with PatchMaster (HEKA) software. Online filtering was achieved using the Bessel $2.9 \mathrm{kHz}$ filtering with a sampling interval of $20 \mathrm{kHz}$. Analysis of the passive membrane parameters was done using a voltage-step protocol as described by D'Angelo et al. (1995).

\section{Evoked Glycinergic Currents}

Coronal cortico-striatal slices were used for recording evoked glycinergic currents from MSNs of $\mathrm{C} 57 \mathrm{Bl} / 6$ mice of two ages (P7 and adults) and GlyR $\alpha 2 \mathrm{KO}$ P5-8 mice. Recordings were done in whole-cell voltage-clamp $(-80 \mathrm{mV}$ holding potential) configuration, using CsCl-based internal solution (in $\mathrm{mM}$ ): $120 \mathrm{CsCl}, 0.022 \mathrm{CaCl}_{2}, 4 \mathrm{MgCl}_{2}, 10 \mathrm{HEPES}, 0.1$ EGTA, 5 phosphocreatine, 4 MgATP, $0.5 \mathrm{Na}_{2} \mathrm{GTP}, 5$ lidocaine N-ethyl chloride and $0.5 \%$ biocytin; $\mathrm{pH}$ 7.2. $10 \mu \mathrm{M} \mathrm{GABA}_{A}$ receptor blocker gabazine (Sigma-Aldrich), $10 \mu \mathrm{M}$ AMPA receptor blocker CNQX, $0.1 \mu \mathrm{M}$ nicotinic ACh receptor blocker $\mathrm{DH} \beta \mathrm{E}$, $5 \mu \mathrm{M}$ NMDA receptor blocker L-689,560 and $1 \mu \mathrm{M}$ voltagegated sodium channel blocker TTX (other substances: Tocris) were present in aCSF. In case of GlyR $\alpha 2 \mathrm{KO}$ mice, gabazine was omitted, and $3 \mathrm{mM}$ GABA was used as additional control for barrel tube position and cell viability. Increasing concentrations of agonists or $\mathrm{KCl}$ (positive control) were applied using a fastperfusion system (SF-77B, Warner Instruments) for a duration of $10 \mathrm{~s}$.

\section{Synaptic and Tonic Glycinergic Currents}

The presence or absence of synaptic glycinergic currents was assessed by measuring mIPSCs in the presence of $10 \mu \mathrm{M}$ gabazine (with CsCl-based internal solution and inhibitors of AMPA,
NMDA receptors, and Na channels, as described below). The presence or absence of tonic glycinergic currents was evaluated by a change in holding current of the cell, patched with CsCl-based internal solution and held at $-80 \mathrm{mV}$ without any inhibitors, upon application of $1 \mu \mathrm{M}$ strychnine. After the baseline period of $1 \mathrm{~min}$, strychnine was applied using a fast-perfusion system, and the holding current was recorded for another $5 \mathrm{~min}$. Mean values of holding current during the first and sixth min of the recording were measured.

\section{Spontaneous Activity and Excitability}

Spontaneous activity and intrinsic excitability of MSNs were evaluated using GlyR $\alpha 2 \mathrm{KO}$ and WT neonatal mice (P6-7). Cells were recorded in perforated-patch or whole-cell currentclamp configurations. In these experiments, we used $30^{\circ}$ tilted horizontal slices for preserving cortico-striatal connections (Jiang and North, 1991). For perforated patches, the pipette solution was composed of (in $\mathrm{mM}$ ): $125 \mathrm{KMeSO}_{3}, 13 \mathrm{KCl}, 10 \mathrm{HEPES}$, $140 \mu \mathrm{g} / \mathrm{ml}$ gramicidin. Measurements started after evaluating the access resistance in voltage-clamp mode by use of $10 \mathrm{mV}$ steps in holding potential starting from $-70 \mathrm{mV}$ (Akaike, 1996). RMP and spontaneous firing were recorded in current clamp configuration without any background current injected. For the whole-cell current-clamp configuration, the pipette solution was composed of (in $\mathrm{mM}$ ): $116 \mathrm{~K}$-gluconate, $15 \mathrm{KCl}$, $5 \mathrm{NaCl}, 10 \mathrm{HEPES}, 4 \mathrm{MgATP}$, and $0.5 \mathrm{Na}_{2}$ GTP. Membrane potential was recorded for $5 \mathrm{~min}$ without any background current injection. For evaluating of the active membrane properties and intrinsic excitability, cells were held at $-70 \mathrm{mV}$, and depolarizing current steps with $10 \mathrm{pA}$ increments were applied, starting from the background current. Individual action potential (AP) analysis was done as described by Chand et al. (2015). Briefly, for individual AP analysis, the first and last AP spike from the train were excluded (generated at $20 \mathrm{pA}$ current injected from rheobase). Average phase plane plots ( $\mathrm{dV} / \mathrm{dt}$ vs. $\mathrm{V})$ were generated from these traces. The slope and rise of the AP was measured by the linear fit to the phase plane plot. Spike width was measured at the midpoint between voltage threshold and maximum voltage.

\section{Inward Rectifying Potassium Currents and Depolarization-Activated Potassium Currents}

Recordings were done in the presence of $1 \mu \mathrm{M}$ TTX. Both hyperpolarizing and depolarizing experiments were recorded in whole-cell voltage-clamp mode. Pipette solution was composed of (in $\mathrm{mM}$ ): $116 \mathrm{~K}$-gluconate, $15 \mathrm{KCl}, 5 \mathrm{NaCl}, 10 \mathrm{HEPES}, 4$ MgATP, and $0.5 \mathrm{Na}_{2} \mathrm{GTP}$. Steps of a $10 \mathrm{mV}$ (for $1 \mathrm{~s}$ ) were used in a range of $-150 \mathrm{mV}$ to $30 \mathrm{mV}$ (from $-70 \mathrm{mV}$ baseline holding potential). Currents were measured as the average amplitude from baseline during the last $50 \mathrm{~ms}$ of each step. Currents were further normalized for cell capacitance which was measured by a voltage-step protocol as described by D’Angelo et al. (1995).

\section{Miniature Excitatory and Inhibitory Postsynaptic Currents (mEPSCs and mIPSCs)}

AMPA-mEPSCs, NMDA-mEPSCs, and mIPSCs were recorded in MSNs of GlyR2 $\alpha \mathrm{KO}$ and WT mice in a whole cell voltage clamp 
configuration at $-80 \mathrm{mV}$. For mEPSCs, intracellular solution contained (in mM): $115 \mathrm{KMeSO}_{3}, 7 \mathrm{KCl}, 0.022 \mathrm{CaCl}_{2}, 4 \mathrm{MgCl}_{2}$, 10 HEPES, 0.1 EGTA, 5 phosphocreatine, 4 MgATP, $0.5 \mathrm{Na}_{2} \mathrm{GTP}$, and $0.5 \%$ biocytin; $\mathrm{pH}$ 7.2. AMPA-mEPSCs were recorded in the presence of $10 \mu \mathrm{M}$ gabazine, $0.1 \mu \mathrm{M}$ DH $\beta E, 5 \mu \mathrm{M}$ L689,560 and $1 \mu \mathrm{M}$ TTX. For NMDA-mEPSCs, L-689,560 was excluded and $10 \mu \mathrm{M}$ of CNQX was added to modified ACSF. For mIPSCs, we used a high chloride intracellular solution, the same as for recordings of evoked glycinergic currents. For mIPSC recording, gabazine was omitted and $10 \mu \mathrm{M} C N Q X$ was added into aCSF. Data were analyzed using the MiniAnalysis program (Synaptosoft).

\section{Peak-Scaled Non-stationary Fluctuation Analysis (NSFA)}

Non-stationary fluctuation analysis (NSFA) was performed from an ensemble of mEPSCs at a holding potential of $-80 \mathrm{mV}$. Events were carefully selected for analysis by visual inspection based on the following criteria: fast rise time to allow for precise alignment, stable baseline, and exponential decay. To estimate the "open probability at peak" and the "number of channels at peak" of AMPARs the MiniAnalysis program (Synaptosoft) was used (Hartveit and Veruki, 2007; Lambot et al., 2016). An averaged mEPSC event was constructed aligning the individual events by their point of maximal rise. The average response waveform was scaled to the peak of individual responses and the variance of the fluctuation of the decay around the mean was calculated. The variance, in 100 bins of equal decrement, was plotted against the mean current amplitude of the decay. The parabolic relationship was fitted by following equation:

$$
\sigma^{2}(\mathrm{I})=\mathrm{iI}-\mathrm{I}^{\wedge} 2 / N+b
$$

, where i was the mean single-channel AMPAR current, I the mean current, $\mathrm{N}$ the number of channels activated at the peak, and $b$ the baseline variance. Parameter $i$ was estimated as the slope of the linear fit of the first portion of the parabola because the equation becomes linear when AMPAR open probability gets close to zero. The number of open channels at the peak was calculated by dividing the average mEPSCs amplitude by the unitary current $\mathrm{i}$.

\section{Evoked Excitatory Postsynaptic Currents (eEPSCs)}

Glutamatergic responses in MSNs of GlyR $\alpha 2 \mathrm{KO}$ and WT mice were evoked by electrical stimulation of corpus callosum by a concentric electrode. Recordings were made from parasagittal slices. Stimulation intensity was selected based upon an input output protocol and set to the intensity that evoked eEPSCs with an amplitude of $50 \%$ of the maximum eEPSCs. For every part of the input - output protocol, 10-20 sweeps were recorded with $30 \mathrm{~s}$ interval time and then averaged during subsequent analysis. Ten micromolars gabazine (Sigma-Aldrich) and $1 \mu \mathrm{M}$ CGP 35348 (Tocris) were added to the aCSF to block GABA $_{A}$ and $\mathrm{GABA}_{B}$ receptors, respectively. For the strychnine condition, $1 \mu \mathrm{M}$ strychnine (Sigma-Aldrich) was additionally added to the recovery aCSF and aCSF. Intracellular solution used was as follows (in mM): $110 \mathrm{CsMeSO}_{3}, 7 \mathrm{KCl}, 0.022 \mathrm{CaCl}_{2}, 4$ $\mathrm{MgCl}_{2}$, $10 \mathrm{HEPES}, 0.1$ EGTA, 5 phosphocreatine, $4 \mathrm{MgATP}$,
$0.5 \mathrm{Na}_{2} \mathrm{GTP}, 5$ lidocaine N-ethyl chloride, and $0.5 \%$ biocytin; $\mathrm{pH}$ 7.2. The paired-pulse ratio was determined after giving two stimulations with $50 \mathrm{~ms}$-interval, and dividing the amplitude of the second peak by the amplitude of the first peak. AMPA/NMDA ratios were estimated by measuring eEPSCs at different holding potentials. Glutamatergic currents were recorded at $-70 \mathrm{mV}$ and $+40 \mathrm{mV}$; after that $5 \mu \mathrm{M}$ NMDA receptor blocker L-689,560 was added to aCSF to isolate AMPAR current at $+40 \mathrm{mV}$. NMDAR currents were calculated by subtracting the response recorded in the presence of $\mathrm{L}-689,560$ at $+40 \mathrm{mV}$, from the response recorded at $+40 \mathrm{mV}$ without this inhibitor. The AMPA/NMDA ratio was calculated by division of the amplitude of AMPAR-mediated response at $-70 \mathrm{mV}$ by the amplitude of NMDAR-mediated response.

\section{Behavioral Tests}

\section{Ultrasonic Vocalizations (USVs)}

Ultrasonic vocalizations (USVs) were evaluated in WT and GlyR $\alpha 2 \mathrm{KO}$ male neonatal pups at P4, P6, and P8. On each day of testing, pups were removed from the nest and placed in a clean empty plastic container located inside a sound-attenuating Styrofoam box. Room temperature was maintained at $22 \pm 1^{\circ} \mathrm{C}$. Recordings were performed using an Avisoft UltraSoundGate condenser microphone capsule CM16 with frequency range $10-180 \mathrm{kHz}$ (Avisoft Bioacoustics, Germany), placed through a hole in the Styrofoam box approximately $20 \mathrm{~cm}$ above the pup. Vocalizations were recorded for $3 \mathrm{~min}$ with a sampling rate of $250 \mathrm{kHz}$ and 16 bit format using Avisoft-RECORDER software V3.2. After testing, pups were weighed and returned to the nest. After testing on P4, pups were also individually identified with a paw tattoo. Acoustic analysis of audio files was performed in Avisoft SASLab Pro V4.4. Spectrograms were generated with a Fourier transformation length of 1024 and time window overlap of $75 \%$ (100\% frame, Hamming window). A high pass filter at a cut-off frequency of $15 \mathrm{kHz}$ was applied to remove background noise outside the relevant frequency band. Call detection was by an automatic threshold-based algorithm and a hold-time mechanism (hold time $20 \mathrm{~ms}$ ). An experienced user blinded to genotype checked accuracy of call detection to ensure concordance between automated and observational detection. Parameters extracted for each time point included mean number of calls/min, mean duration of calls, and quantitative analyses of sound frequencies.

\section{Neonatal Righting Reflex}

Neonatal righting reflex was assessed in P5, P6, and P7 WT and GlyR $\alpha 2 \mathrm{KO}$ male littermates. Briefly, pups were placed in a horizontal recumbent position on a cleaned flat surface free of obstacles. The time pups needed to right onto all fours was measured. If pups did not right after $1 \mathrm{~min}$, a time of $60 \mathrm{~s}$ was recorded, and the test was stopped. Each pup was evaluated three times per experimental day.

\section{RNA and Protein Isolation}

Animals at different developmental stages were anesthetized and decapitated for brain extraction. Brains slices of $300 \mu \mathrm{m}$ were prepared on a vibratome (Leica VT1000S) in RNase-free 0.01 M 
PBS solution. Dorsal striatum was dissected for RNA extraction. For qPCR and Western blotting, RNA and proteins were isolated simultaneously by guanidinium thiocyanate-phenolchloroform (AGPC) method using QIAzol lysis reagent (Qiagen, Germany). Briefly, samples were homogenized in QIAzol and the RNA was separated to an upper phase whilst the proteins remained in the lower phase. The upper phase was collected and further decontaminated by a second chloroform step. Subsequently, the RNA and proteins were precipitated separately and washed according to the QIAzol protocol. The quality and quantity of the RNA was accessed using the NanoDrop 1000 (Thermo Fisher Scientific). Synthesis of cDNA was performed by High Capacity cDNA Reverse Transcription Kit (Applied Biosystems $^{\mathrm{TM}}$, Thermo Fisher Scientific, Belgium). The protein pellet was dissolved in $100 \mu \mathrm{l} 1 \%$ sodium dodecyl sulphate (SDS) supplemented with Pierce ${ }^{\mathrm{TM}}$ Protease Inhibitor Mini Tablets (Thermo Fisher Scientific) and stored at $-20^{\circ} \mathrm{C}$. The protein concentration was determined by bicinchoninic acid assay (Pierce BCA protein assay kit, Thermo Fisher Scientific).

\section{RT-QPCR and Analysis}

cDNA amplification was performed using Fast SYBR green master mix (Applied Biosystems) containing $3 \mathrm{mM}$ forward and $3 \mathrm{mM}$ reverse oligonucleotide primers (Table 1), RNAse-free water, and $12.5 \mathrm{ng}$ cDNA template in a total reaction volume of $10 \mu \mathrm{l}$. Universal cycling conditions were used $\left(95^{\circ} \mathrm{C}\right.$ for $20 \mathrm{~s}, 40$ cycles of $95^{\circ} \mathrm{C}$ for $3 \mathrm{~s}$, and $60^{\circ} \mathrm{C}$ for $30 \mathrm{~s}$ ) (Jiang and North, 1991). Specificity of PCR products was determined using melting curve analysis (StepOne Software V2.3) and molecular weight analysis ( $2 \%$ agarose gel) following the amplification protocol. Efficiency of PCR products was evaluated using standard curves of pooled samples. Data were normalized against the most stable reference genes as determined by qBasePlus software (Biogazelle). Relative quantification of gene expression was performed using the comparative $2^{-\Delta \Delta C t}$ method (Pfaffl, 2001). Expression levels were converted to fold change values for gene expression and compared to the averaged wild-type value of the youngest tissue samples tested for that specific gene, which was set to one.

\section{Western Blotting}

Isolated and dissolved proteins were mixed with sample buffer (5:1, v/v) containing $300 \mathrm{mM}$ TrisHCl (pH 6.8), 6\% SDS,

TABLE 1 | Sequence and supplier information of primers used.

\begin{tabular}{|c|c|c|}
\hline Gene & Forward primer & Reverse primer \\
\hline RPL 13A & $\begin{array}{l}\text { 5'-CTGGTACTTCC ACCCG } \\
\text { ACCTC-3' }\end{array}$ & $\begin{array}{l}\text { 5'-GGATCCCTCCACCCTAT } \\
\text { GACA-3' }\end{array}$ \\
\hline$P G K 1$ & $\begin{array}{l}\text { 5'-GAAGGGAAGGGAAA } \\
\text { AGATGC-3' }\end{array}$ & $\begin{array}{l}\text { 5'-GCTATGGGCTCGGT } \\
\text { GTGC-3' }\end{array}$ \\
\hline Gene & Supplier & Cat. No. \\
\hline GLRA1 & Qiagen & QT00172221 \\
\hline GLRA2 & Qiagen & QT00132020 \\
\hline GLRA3 & Qiagen & QT01657943 \\
\hline$G L R B$ & Qiagen & QT00162911 \\
\hline
\end{tabular}

$30 \%$ glycerol, $15 \% \beta$-mercaptoethanol, and $0.01 \%$ bromophenol blue. Samples containing $20 \mu \mathrm{g}$ protein were separated by $10 \%$ SDS polyacrylamide gel electrophoresis (SDS-PAGE) in Mini-PROTEAN systems (Bio-Rad) and transferred onto polyvinylidene fluoride (PVDF) membranes. Membranes were blocked with $5 \%$ BSA in Tris-buffered saline containing $0.1 \%$ Tween-20 (TBS-T) for $1 \mathrm{~h}$ at room temperature. The blots were probed with primary antibody, rabbit anti-PSD95 (1:1000; ab18258, Abcam) and guinea-pig anti-VGluT1 (1:1000 ab5905, Millipore) in blocking solution overnight at $4^{\circ} \mathrm{C}$. $\beta$-Actin was used as a reference. The membranes were incubated for $1 \mathrm{~h}$ at RT with subsequent HRP-conjugated secondary antibodies in TBST. Signal was evoked using Pierce ECL 2 Substrate (Thermo Fisher Scientific). Bands were visualized by chemiluminescent imaging (ImageQuant ${ }^{\mathrm{TM}}$ LAS $4000 \mathrm{mini}$ ). Analyses were performed utilizing 16-bit scaled intensity measurements of target, reference and background areas in FIJI ImageJ (Schindelin et al., 2012). Results were compared to the averaged value of P7 WT samples, which was set to one.

\section{Data Analysis and Statistics}

Curve fitting and data analyses were performed with Igor Pro 6.0 (WaveMetrics) and GraphPad Prism 7. Histogram and box plots were used for graphic representation. On the histograms, mean with SEM are shown. On the box plot, the central line represents the median, the edges represent the interquartile ranges, and the whiskers represent the overall distribution. The D'AgostinoPearson omnibus normality test was used to determine a normal distribution. Pairwise comparisons were performed on normal distribution using unpaired or paired $t$-test (unless specifically otherwise mentioned), as appropriate; non-normal distributions were analyzed by a Mann-Whitney $U$ test. Some data were analyzed by a one-way repeated-measures ANOVA followed by a Tukey's multiple comparison post hoc test or by a twoway repeated-measures ANOVA followed by Bonferroni multiple comparisons post hoc test, depending on the experimental design. The level of significance was established as follows: ${ }^{*} p<0.05$, ** $p<0.01$, and ${ }^{* * *} p<0.001$.

\section{RESULTS}

\section{The GlyR $\alpha 2$ Subunit in MSNs Is Downregulated During Postnatal Development}

We have shown previously that MSNs of adult mice express functional GlyRs with $\alpha 2$ being the main agonist-binding subunit (Molchanova et al., 2018). To assess the developmental role of GlyRs in the striatum, the expression pattern of the different GlyR subunits was first identified in the developing DS. Quantitative real-time PCR on microdissected DS of mice at different ages showed that the expression of Glra2 is developmentally downregulated during maturation (Figure 1A). However, Glra1, Glra3, and Glrb did not exhibit a developmental change in their expression levels (Figure 1A) and the overall expression of Glra1 and Glra3 was around 20-times lower than for Glra2 
A
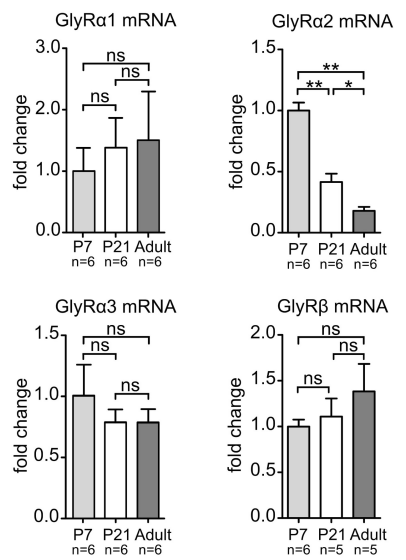

D

P5-P8 WT MSN

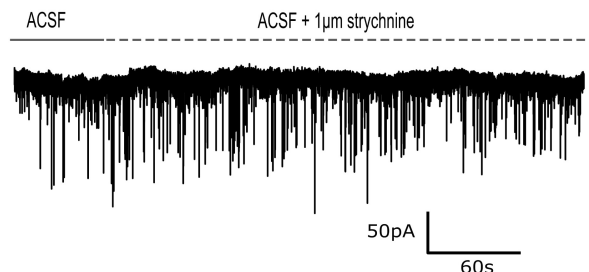

G

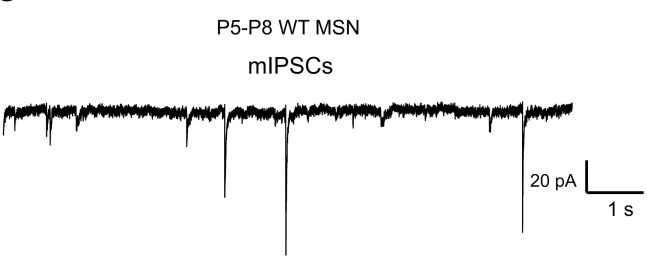

B

P5-P8 WT MSN

Adult WT MSN

P5-P8 KO MSN
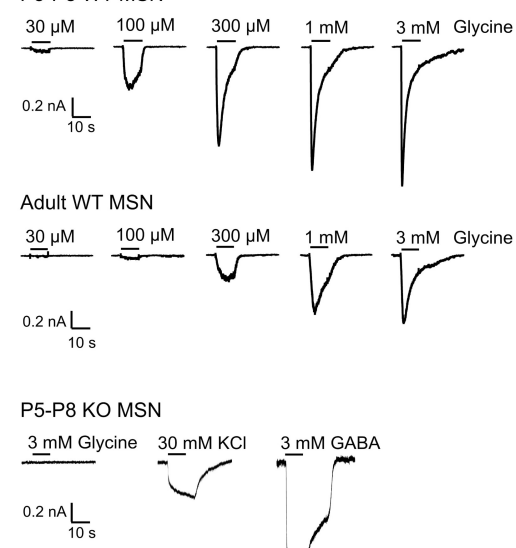

$\int^{3 \mathrm{mM} \mathrm{GABA}}$

E

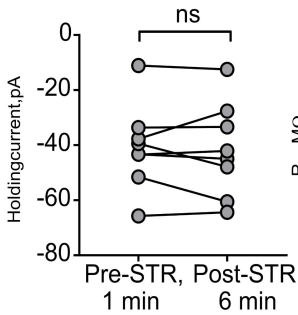

$\mathbf{F}$
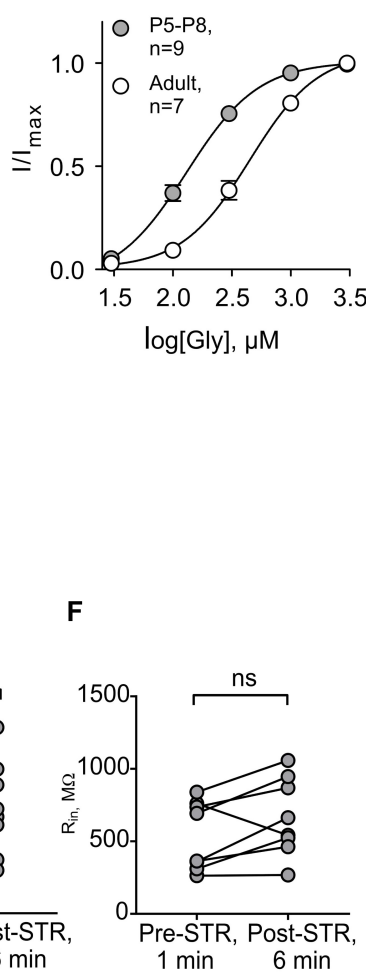

G1

P5-P8 WT MSN

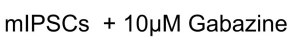

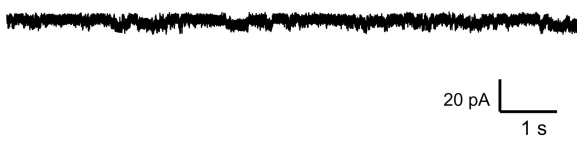

FIGURE 1 | Transcriptional and functional comparison of GlyRs at different developmental stages. The GlyR $\alpha 2$ subunit mRNA is developmentally down-regulated which corresponds to the lower current density (pA/pF) of evoked glycinergic currents in adult dorsal striatum MSNs. (A) qPCR results from dorsal striatum show a stark decrease in the transcription of the GlyR $\alpha 2$ subunit gene $\left({ }^{*} p<0.05 ;{ }^{* *} p<0.01\right.$ ). Data are normalized to the expression at P7 and presented as mean \pm SEM. GlyR $\alpha 1, \alpha 3$, and GlyR $\beta$ subunit mRNAs did not show a developmental up- or down-regulation. (B) Representative dose-response traces from glycine-evoked currents in whole cell. In the P5-P8 GlyRa2KO mice no glycinergic currents were measured in dorsal striatum MSNs $(n=7)$. (C) Relative dose-response curves from neonate (P5-P8) and adult WT MSNs to glycine. (D) Representative trace of a P5-P8 MSN clamped at -80 mV with application of ACSF (1 $\mathrm{min}$ ) and ACSF + STR (5 min). (E,F) values of the holding current (pA) and input resistance (M $\Omega$ ) measured after 1 min of ACSF (Pre-STR) or 5 min after ACSF+strychnine (Post-STR) application in WT P5-P8 MSNs $(n=8)$. (G,G1) Representative traces of phasic GABAergic mIPSCs and (G1) phasic current in the presence of gabazine. mIPSCs were isolated by application of inhibitors, as described in the Section "Materials and Methods."

(Supplementary Figures 1A,B). For Glra4, expression remained undetected and was consequently not included in the results. In fact, Glra1 and Glra3 mRNAs were barely detectable and this finding was confirmed by functional analysis. In both neonatal (P5-8) and adult WT MSNs, glycinergic currents were measured in whole-cell patch-clamp mode, but none of the neonatal MSNs showed a glycinergic response in GlyR $\alpha 2 \mathrm{KO}$ (KO) mice (Figure 1B). This implies that the GlyR $\alpha 2$ subunit is the only ligand binding subunit to be present at functionally relevant expression levels in developing MSNs, similar to adult ones (Molchanova et al., 2018). The maximum current density was also significantly higher in neonatal versus adult MSNs, which might suggest that the function of this glycinergic current changes during development (Table 2). The relative doseresponse curve shows a rightward shift for the adult MSN population as compared to neonatal MSNs (Figure 1C). This correlates with a lower effective concentration $\left(\mathrm{EC}_{50}\right)$ in the neonatal MSNs for glycine as compared to the adult MSNs (Table 2). Together, these data indicate that the GlyR $\alpha 2$ subunit is the only agonist-binding subunit present at all stages of development, and that it has a higher receptor density and affinity in developing MSNs as compared to the adult 
TABLE 2 | Characteristics of evoked glycinergic currents in neonatal and adult MSNs (mean \pm SEM)

\begin{tabular}{lccc}
\hline & $\begin{array}{c}\text { WT MSNs, } \\
\text { P5-P8 }\end{array}$ & $\begin{array}{c}\text { WT MSNs, } \\
\text { adult }\end{array}$ & P-value \\
\hline $\mathrm{EC}_{50}(\mu \mathrm{M})$ & $139.24 \pm 16.87$ & $470.44 \pm 67.15$ & 0.0002 \\
Hill coefficient & $1.52 \pm 0.10$ & $1.63 \pm 0.08$ & 0.2509 \\
Max. current density $(\mathrm{pA} / \mathrm{pF})$ & $22.75 \pm 3.69$ & $5.54 \pm 1.60$ & 0.0021 \\
$N$ & 9 & 7 & \\
\hline
\end{tabular}

MSNs, highlighting its importance for developing striatal MSN population.

\section{At P7, No Glycinergic Synaptic or Extrasynaptic Currents Are Present in Neostriatal MSNs}

While we established the presence and characteristics of the evoked glycinergic currents in neonatal MSNs, their physiological function remains unidentified. Tonic and phasic glycinergic signaling depends on receptor localization, i.e., extrasynaptic and synaptic, respectively. In adult dorsal MSNs, endogenous glycinergic currents have been found to work via tonic receptor activation (Molchanova et al., 2018). However, considering this fact, it is well known that these processes are often developmentally regulated and thus agedependent (Valtcheva et al., 2017). Using a high-chloride filling solution in the whole-cell configuration we tested for the presence of both types of glycinergic signaling. In neonatal MSNs, at P6-P8, no significant change in holding current was detected in WT animals upon strychnine application (Figures 1D-F; IHold Pre-STR: $-40.74 \pm 5.497$ pA vs. PostSTR: $-41.68 \pm 6.034 \mathrm{pA})$. The same outcome was observed in MSNs of the GlyR $\alpha 2 \mathrm{KO}$ animals (Supplementary Figures 1C,D). Furthermore, when all ionotropic glutamate and GABA receptors where pharmacologically blocked, in the presence of TTX, no currents could be detected indicating of the absence of functional synaptic GlyRs (Figures 1G,G1). This strongly suggests that there is no tonic or phasic glycinergic signaling in neonatal dorsal MSNs.

\section{Spontaneous and Evoked Action Potential Firing Is Impaired in Neonatal GlyR $\alpha 2 \mathrm{KO}$ Mice}

Early postnatal spontaneous activity has been described as a mechanism underlying the development of neurons and synapses for many brain structures, including dorsal striatum (Kerschensteiner, 2014). Since agonist-gated chloride channels, such as $\mathrm{GABA}_{A}$ Rs and GlyRs, play a major role in initiation and propagation of neonatal activity (Ben-Ari, 2002), we evaluated the role of GlyRs in action potential firing of MSNs in P6-7 mice. Spontaneous and evoked action potential firing was recorded in wild-type and GlyR $\alpha 2 \mathrm{KO}$ MSNs using the perforated patchclamp method in order to minimally influence the intracellular environment (Vogt, 2015). In the dorsal striatum of WT animals, 11 out of 35 cells ("active" MSNs, 31\%) fired spontaneous action potentials (Figure 2A), when recorded in current-clamp mode without any background current injection. These spontaneously active cells seem to be less developed, than silent cells, based on the firing frequency of the APs, evoked by depolarizing steps, and RMP (Supplementary Figure 2). Spontaneously active MSNs displayed an irregular firing pattern, and no bursting activity was observed. In GlyR $\alpha 2 \mathrm{KO}$, a similar fraction of MSNs was spontaneously active (Figure 2A; 6 out of 17 cells, $35 \%$; $p=0.8$ by Fisher's test). Quantification of the spontaneous firing frequency (Figure 2B) indicated that the frequency of action potentials was significantly lower in the GlyR $\alpha 2 \mathrm{KOs}(1.08 \pm 0.31 \mathrm{~Hz})$ as compared to the WT MSNs $(2.37 \pm 0.43 \mathrm{~Hz} ; p<0.05)$. This change in firing frequency was not accompanied by a significant change in the membrane potential (Figure 2C), and passive membrane properties, such as input resistance and cell capacitance remain unaffected (Table 3). To rule out NMDA mediated component of spontaneous activity, we recorded NMDA-mEPSCs. At this developmental stage, NMDA-receptors are of the NR2C/D type which are known to have longer decay time and are crucial for synapse-driven calcium events in immature MSNs (Dehorter et al., 2011). No significant differences were found in NMDA-mEPSCs in WT versus GlyR $\alpha 2 \mathrm{KOs}$ for either frequency (WT: $0.26 \pm 0.02 \mathrm{~Hz}, \mathrm{KO}: 0.22 \pm 0.01 \mathrm{~Hz}$ ) or amplitude (WT: $16.45 \pm 0.75 \mathrm{pA}$, KO: $15.97 \pm 0.70 \mathrm{pA}$ ) of the events (Supplementary Figure 3).

To further evaluate the intrinsic excitability of spontaneously active neonatal DS MSNs, current was injected intrasomatically in the current-clamp mode, starting from membrane potential values of $-70 \mathrm{mV}$ (Figure 2D). In both WT and GlyR $\alpha 2 \mathrm{KO}$ MSNs, first APs were evoked at similar threshold and rheobase values (Table 3). Upon increase of the values of injected current, WT and GlyR $\alpha 2 \mathrm{KO}$ cells fired APs with similar frequencies (Figure 2F). However, in GlyR $\alpha 2 \mathrm{KO}$ MSNs, APs started to accommodate at significantly lower values compared to wild-type MSNs (Figure 2E, $68 \pm 4.90 \mathrm{pA}$ in KOs vs. $117 \pm 14.07 \mathrm{pA}$ in WTs; $p<0.05)$.

To explain the decreased spontaneous firing frequency and accommodation current in GlyR $\alpha 2 \mathrm{KO}$, an individual AP analysis was performed (Figure 2G). Both the amplitude and duration of APs in spontaneously active GlyR $\alpha 2 \mathrm{KO}$ cells were severely affected. In GlyR $\alpha 2$ KO DS MSNs, the AP halfwidth is higher (Figure $\mathbf{2 H}, 3.20 \pm 0.43 \mathrm{~ms}$ ) as compared to WT DS MSNs $(2.05 \pm 0.08 \mathrm{~ms} ; p<0.05)$. This was accompanied by significant changes in the rise and decay rates (Table 3). The amplitude of the APs differed significantly when comparing "active" KO and WT cells (Figure 2I). No significant changes were found in the after-hyperpolarization parameters (Table 3). We were able to replicate these results when we acutely blocked the GlyR $\alpha 2$ subunit with strychnine: using a modified intracellular solution, we recorded in whole-cell current clamp configuration and evoked action potentials (at rheobase $+20 \mathrm{pA}$ ) in spontaneously active MSNs. In recordings from the same WT cells, the action potential broadened after 5 min application of strychnine (Figures 2J-K, Pre-STR: $1.30 \pm 0.10 \mathrm{~ms}$, Post-STR: $1.48 \pm 0.11 \mathrm{~ms} ; p<0.05)$. Similarly, a significant decrease was also found for the amplitude of the action potential (Figures 2J,L, Pre-STR: $70.33 \pm 3.14 \mathrm{mV}$, 
A

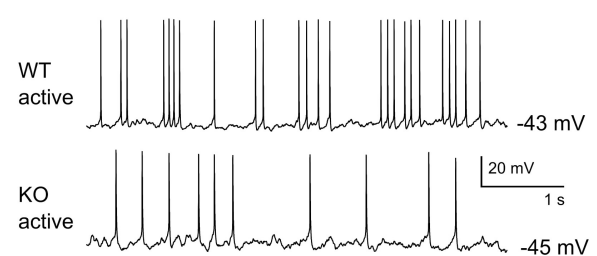

D
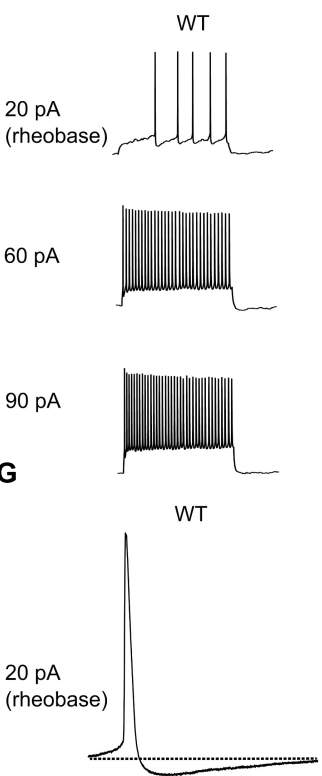

J

WT

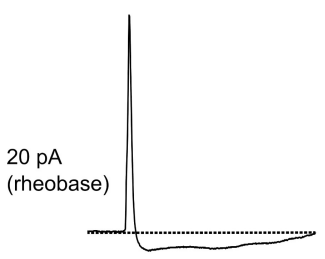

KO

KO
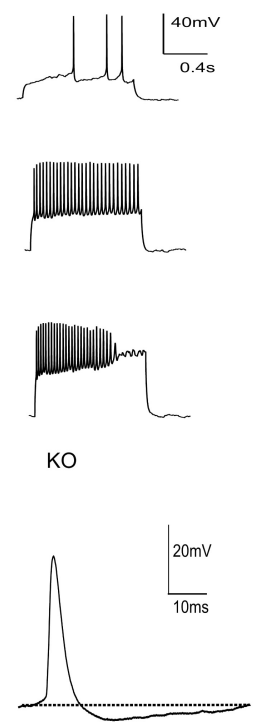

WT + Strychnine

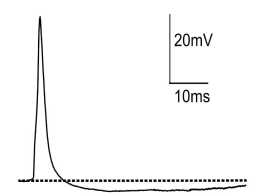

B
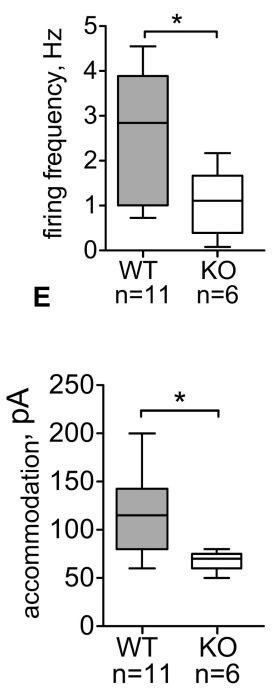

H

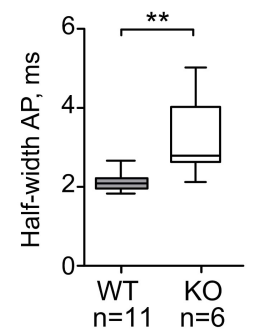

K

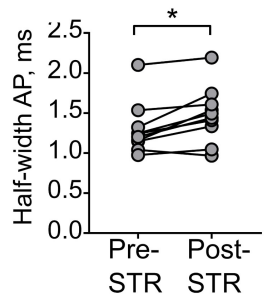

C
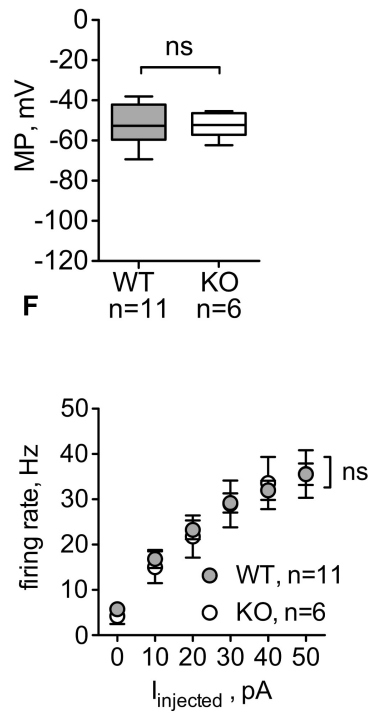

I

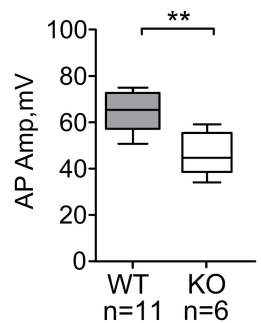

L

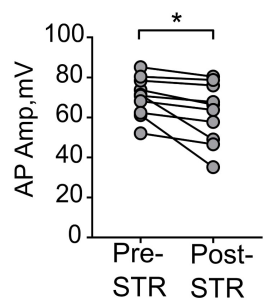

FIGURE 2 | Active neonatal MSNs from GlyRa2KO mice (P6-7) have slower rates of spontaneous firing and altered properties of evoked action potentials. (A) Representative traces from spontaneous activity in WT and KO MSNs. (B) Firing frequency of spontaneous action potentials is significantly reduced in the KO MSNs. (C) Membrane potential of active WT and KO MSNs. (D) Representative traces showing evoked action potentials in active MSNs in WT and KO dorsal striatum. (E) Injected current, needed to evoke accommodation in active WT and KO MSNs. (F) Action potential firing rate as a function of injected current, starting from rheobase. (G) Example traces of individual action potential, recorded in WT and KO MSNs. (H,I) Action potential half-width (ms) and amplitude (mV) of individual action potentials from WT and KO MSNs. (J) Example traces of individual action potentials, recorded in WT MSNs before and after 5 min of $1 \mu M$ strychnine application. $(\mathbf{K}, \mathbf{L})$ Action potential half-width $(\mathrm{ms})$ and amplitude $(\mathrm{mV})$ of individual action potentials recorded in WT and KO MSNs after $1 \mu \mathrm{M}$ strychnine application. Pooled data are presented as either mean \pm SEM or box plot $\left({ }^{*} p<0.05,{ }^{* *} p<0.01\right)$.

Post-STR: $62.14 \pm 4.73 \mathrm{mV} ; p<0.05)$. We furthermore looked at inward rectifying currents and potassium currents activated by depolarization in P7 "active" WT and KO MSNs and P14 WT and KO MSNs (no spontaneous APs were seen in P14 MSNs). At P7 we found significantly lower inward rectifying currents (Figure 3B) for the voltage steps $-150,-140$, and $-130 \mathrm{mV}$ (respectively, pA/pF; WT: $-11.025 \pm 3.43 \mathrm{KO}:-2.068 \pm 0.5$; WT: $-9.07 \pm 2.72 \mathrm{KO}:-1.833 \pm 0.45$; WT: $-7.777 \pm 2.72$
KO: $-1.470 \pm 0.36)$. This was, however, not observed in the more developed P14 MSNs (Figure 3D) indicating a transient effect at P7. This reduced functioning of inward rectifying current might be a consequence of either delayed development of the MSNs, or loss of GlyR-mediated modulation of some inward-rectifying potassium channels (Dehorter et al., 2011). No effects were observed on the voltage-gated potassium currents (Figures 3A,C). 
TABLE 3 | Passive membrane properties and intrinsic excitability parameters of active neonatal MSNs from wild-type and GlyR $\alpha 2 \mathrm{KO}$ animals (mean $\pm \mathrm{SEM}$ ).

\begin{tabular}{|c|c|c|c|}
\hline & $\begin{array}{c}\text { Active MSNs, } \\
\text { WT P6-7 }\end{array}$ & $\begin{array}{c}\text { Active MSNs, } \\
\text { GlyR } \alpha 2 \text { KO P6-7 }\end{array}$ & $P$-value \\
\hline Input resistance (M $\Omega$ ) & $1555 \pm 296.8$ & $1975 \pm 496.1$ & 0.403 \\
\hline Capacitance (Cm) & $89.55 \pm 17.43$ & $53.88 \pm 8.322$ & 0.385 \\
\hline Rheobase (pA) & $24 \pm 5.812$ & $18.33 \pm 3.073$ & 0.821 \\
\hline Threshold (Vm) & $-40.05 \pm 3.385$ & $-39.37 \pm 1.679$ & 0.727 \\
\hline Ihold to -70 mV (pA) & $-17.55 \pm 4.764$ & $-13.83 \pm 2.686$ & 0.862 \\
\hline Rate of AP rise (mV/ms) & $150.80 \pm 17.30$ & $62.72 \pm 10.94$ & 0.002 \\
\hline Rate of AP decay (mV/ms) & $30.76 \pm 1.88$ & $15.38 \pm 2.52$ & 0.001 \\
\hline Fast AHP, half-width (ms) & $25.86 \pm 1.951$ & $23.39 \pm 2.195$ & 0.097 \\
\hline Fast AHP, amplitude (mV) & $5.468 \pm 0.215$ & $6.109 \pm 0.387$ & 0.802 \\
\hline$N$ & 11 & 6 & \\
\hline
\end{tabular}

These data indicate that GlyR $\alpha 2$ affects the spontaneous activity in neonatal MSNs and, when pharmacologically blocked or genetically ablated, cause the broadening of the AP waveform and reduced frequency of spontaneously occurring APs in "active" cells, without affecting the passive membrane properties or after-hyperpolarizing potentials. Probably, the effect of GlyR $\alpha 2$ is mediated by some inward rectifying potassium channel. Interestingly, we did not find any difference in passive membrane properties and intrinsic excitability properties when comparing "silent" neonatal MSNs from WT and GlyR $\alpha 2 \mathrm{KO}$ animals (Supplementary Table 1). Since "silent" cells seem to be functionally more developed, than "active" cells, it appears that the impact of GlyRs on the firing properties of MSNs is only present at certain developmental stages and disappears with maturation of the cell. However, the role of GlyRs in AP firing of neonatal MSNs may have dramatic consequences on the density of synaptic connections in adult animals.

\section{Functional Glutamatergic Innervation Is Reduced in GlyR $\alpha 2 \mathrm{KO}$ Animals}

Proper glutamatergic innervation is essential for the MSNs to overcome the strong inhibitory inputs received from other MSNs and interneurons and thus convey cortical and thalamic signals to downstream motor nuclei (Surmeier et al., 2007). Both genetic factors (Chen et al., 2016) and intrinsic activity (Huupponen et al., 2013) fine tune the development of glutamatergic synapses and here we evaluated the contribution of GlyR $\alpha 2$ in functional maturation of the glutamatergic innervation of MSNs. First, we recorded AMPA-mEPSCs at different developmental stages (P7, P14 and adults) (Figure 4A). In all developmental stages, we observed a reduction of the frequencies of mEPSCs (Figure 4B). The amplitude of these events was not affected (Figure 4C). This implies that number of functional glutamatergic synapses is smaller in the GlyR $\alpha 2$ KO DS MSNs without affecting the NMDA (frequency and amplitude) component of the glutamatergic transmission (Supplementary Figure 3). GABAergic synaptic transmission was not affected by deletion of Glra2, since the properties of mIPSCs were unchanged in adult GlyR $\alpha 2 \mathrm{KO}$ MSNs as compared to WT (Supplementary Figure 4). To rule out the acute contribution of a direct modulation of glutamatergic transmission by GlyRs, we evaluated the acute effect of GlyR antagonist strychnine $(1 \mu \mathrm{M})$ on mEPSCs recorded from DS MSNs of WT adult mice. Application of strychnine did not cause any reduction in the frequency and amplitude of mEPSCs (Figures 4D-F). In addition, the single synaptic parameter of open channels at peak of the averaged mEPSC event was evaluated by non-stationary fluctuation analysis (Figure 4G). The number of open channels was reduced throughout development suggesting an impaired development of AMPA-mediated mEPSCs. In conclusion, these data demonstrate that the number of functional glutamatergic synapses onto GlyR $\alpha 2 \mathrm{KO}$ MSNs is reduced from early postnatal development and persists into adulthood. Notably, this reduction is not due to a direct effect of glycinergic transmission because strychnine application was not able to reproduce this. To see if reduced glutamatergic input onto the MSNs would be seen in motoric changes we used the righting reflex test. Disturbed motor behavior was observed in postnatal right reflex, measured as the time to right on all four paws. This was significantly slower in GlyR $\alpha 2 \mathrm{KO}$ pups starting at P6 (Figure 4I). In the mouse, the motor regions of the brain, including the striatum, are activated during vocal production (Arriaga et al., 2012) due to intrinsic MSN activity and glutamatergic connectivity originating from the cortex to the MSNs (Kim et al., 2012; Araujo et al., 2015). The rate of neonatal vocalization (mean calls/min) upon isolation from the nest is significantly increased at P8 in GlyR $\alpha 2 \mathrm{KO}$ compared to WT controls, but not at earlier time points (Figure $4 \mathbf{H}$ ). This suggests that the developmental decrease in vocalization that normally occurs after peak vocalization at P6-P7 (Scattoni et al., 2008; Ey et al., 2013; Araujo et al., 2015) is delayed in GlyR $\alpha 2 \mathrm{KO}$ mice. To explain these findings, we investigated the pre- and postsynaptic molecular and morphological characteristics of synapses on MSNs in WT and GlyR $\alpha 2 \mathrm{KO}$ mice.

\section{The Number of Glutamatergic Synaptic Contacts Is Not Affected by GIra2 Deletion}

Previous reports have demonstrated that the absence of the GlyR $\alpha 2$ subunit causes changes in synaptic density and neuronal ramifications in the cortex (Morelli et al., 2016). Similar processes might contribute to the observed reduction of functional glutamatergic inputs on MSNs in the dorsal striatum. We therefore performed an analysis of the morphology of the DS MSNs in WT and GlyR $\alpha 2 \mathrm{KO}$ mice at different developmental stages. The morphology of the DS MSN dendritic tree was not affected in GlyR $\alpha 2 \mathrm{KO}$ mice of different ages in comparison to WT mice (Supplementary Figures 5A,B). Furthermore, in P7, P21 and adult DS MSNs, no alterations of the spine density were seen for GlyR $\alpha 2 \mathrm{KO}$ mice as compared to WT mice (Figures 5A,B).

Besides morphological changes, expression of synaptic proteins might also be an underlying mechanism for the observation of impaired glutamatergic network formation (Peca et al., 2011). The protein expression of the post-synaptic AMPAR and NMDAR anchoring protein PSD-95, and pre-synaptic corticostriatal vesicular glutamatergic transporter protein 
A

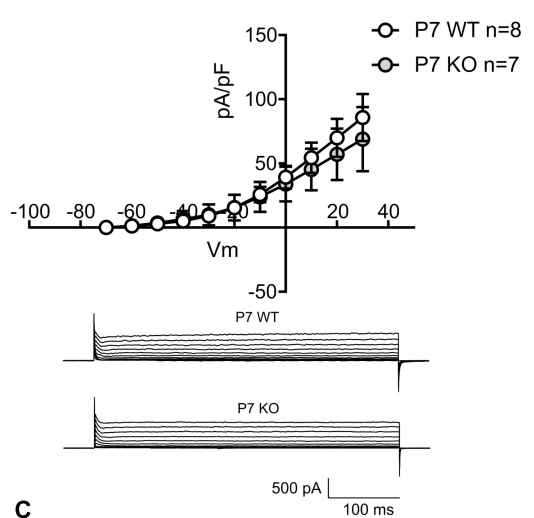

C
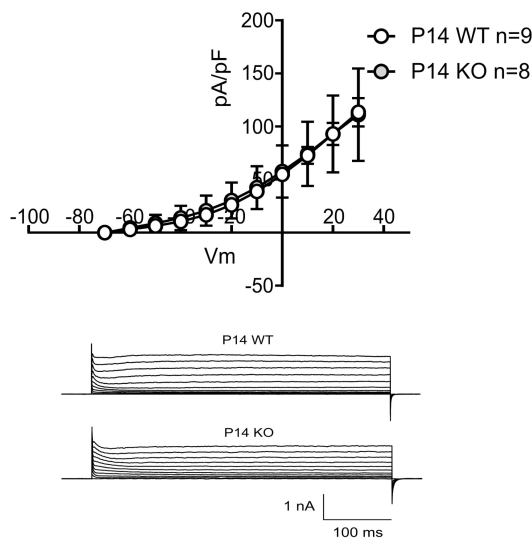

B

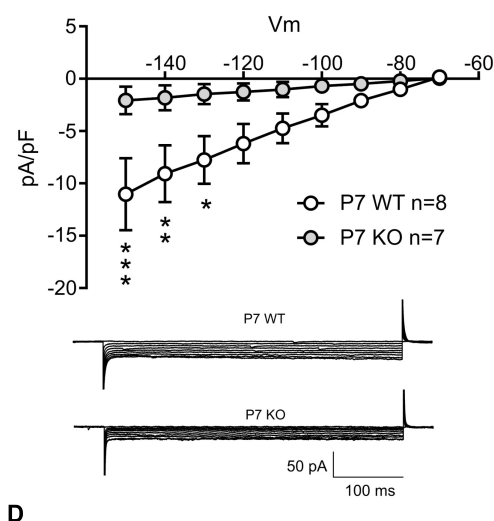

D
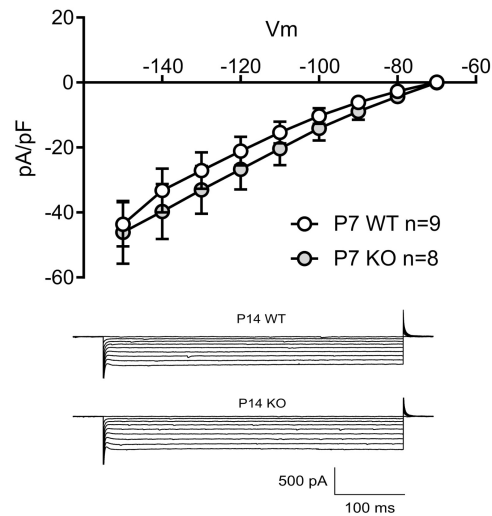

FIGURE 3 | Evaluating the outward and inward potassium currents in P7 active MSNs and P14 silent MSNs in WT and KO mice. (A) Current density plotted for depolarizing membrane potentials in P7 active MSNs in WT and KO, and example traces of outward potassium currents recorded in P7 active MSNs in WT and KO mice. (B) Current density plotted for hyperpolarizing membrane potentials in P7 active MSNs in WT and KO, and example traces of inward potassium currents recorded in P7 active MSNs in WT and KO mice. $\left(n_{W T}=8 ; n_{K O}=7\right)$. (C) Current density plotted for depolarizing membrane potentials in P14 MSNs in WT and KO, and example traces of outward potassium currents recorded in P14 MSNs in WT and KO mice. (D) Current density plotted for hyperpolarizing membrane potentials in P14 MSNs in WT and KO, and example traces of inward potassium currents recorded in P14 MSNs in WT and KO mice. $\left(n_{W T}=9 ; n_{K O}=8\right)$. Data are presented as mean $\pm \operatorname{SEM}\left(* p<0.05,{ }^{* *} p<0.01,{ }^{* * *} p<0.001\right)$.

VGluT1 were evaluated (Berry and Nedivi, 2017; Fleischer et al., 2017). PSD-95 (Figure 5C) was expressed in equal amounts which is indicative of an unaltered number of glutamatergic synapses (Peca et al., 2011). The cortical glutamatergic afferents are also unlikely to be affected in the absence of the GlyR $\alpha 2$ subunit, as was evident from Western blot analysis of VGluT1 (Figure 5D). This suggested that the reduction in functional glutamatergic innervation in GlyR $\alpha 2 \mathrm{KO}$ was unlikely to be a consequence of an impaired morphological development or spine generation of the DS MSNs at any developmental stage we investigated. A more in-depth analysis of the glutamatergic synapse in the dorsal striatal MSNs was therefore essential.

\section{Reduction of the Functional Connectivity May Be Due to Impaired Maturation of Silent Synapses}

Reduction in the frequency of AMPA-mEPSCs in GlyR $\alpha 2 \mathrm{KO}$ MSNs without any morphological changes might be the result of a pre- or postsynaptic defect. Developmental defects of the cortical afferent might lead to a reduced release probability of glutamatergic vesicles that might not be detected by measuring VGluT1 levels. Also, the existence of presynaptically located GlyRs cannot be ruled out. Postsynaptic defects might be the result of early changes in intrinsic activity which would manifest by changes in the AMPA/NMDA ratios. To evaluate these two possibilities, we recorded the paired-pulse ratio (PPR) of AMPAR-mediated evoked excitatory postsynaptic currents (eEPSCs) (Figure 6A) and measured the AMPA/NMDA eEPSC ratio (Figure 6C). No effect on the $\mathrm{PPR}$ was found in either the GlyR $\alpha 2$ KO (PPR: $1.46 \pm 0.05)$ mice or when the GlyRs were pharmacologically blocked (PPR WT: $1.23 \pm 0.08$; WT+STR: $1.26 \pm 0.04$ ) (Figure 6B). Interestingly, we saw a reduction in the AMPA/NMDA-ratio that was only present in GlyR $\alpha 2 \mathrm{KO}$ mice (Figure 6D). No acute effects were detected when GlyRs were pharmacologically blocked (WT: $6.78 \pm 0.60$; WT+STR: $6.68 \pm 0.59$; KO: $3.84 \pm 0.42$ ). In summary, we can state that the post-synaptic reduction in AMPA/NMDA parameter is indicative 
A

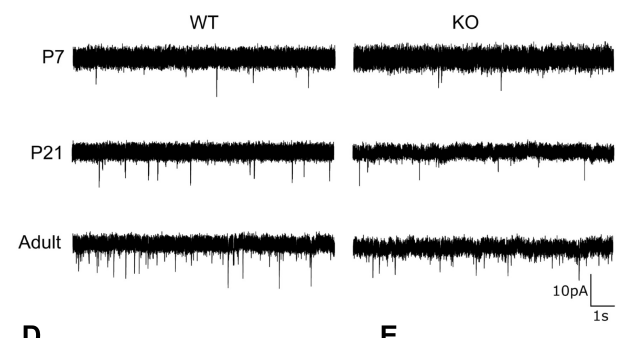

D

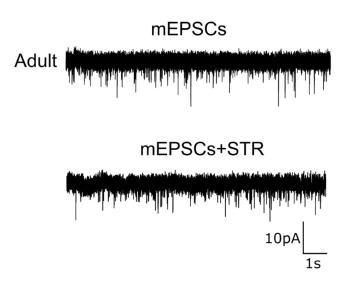

E

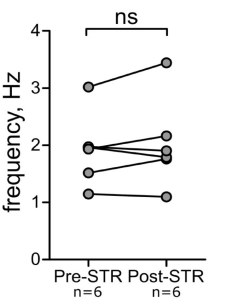

B

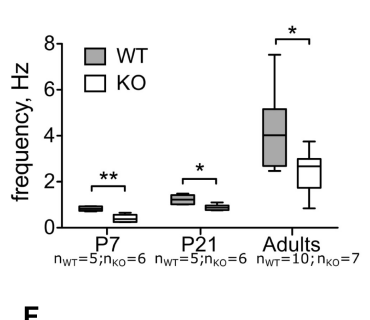

C
H

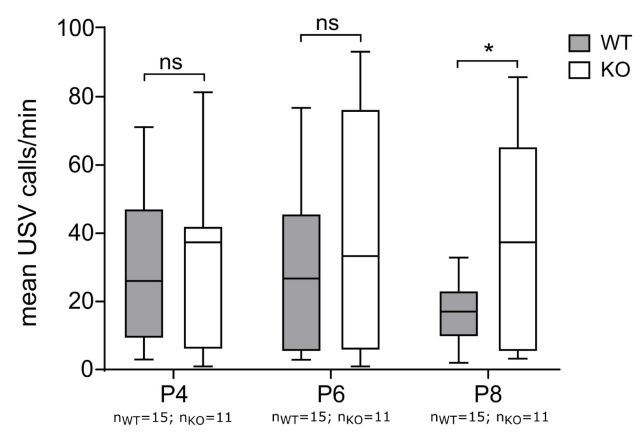

I

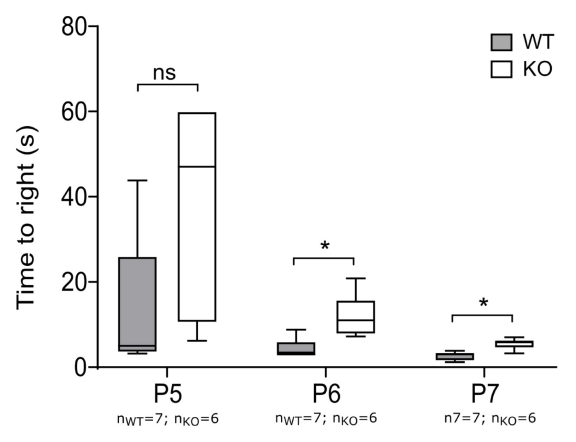

FIGURE 4 | Functional glutamatergic innervation of MSNs is decreased in GlyRa2KO mice. (A) Example traces of mEPSCs in WT and KO MSNs of different ages. (B,C) Frequency and amplitude of mEPSCs in WT and KO MSNs of different ages. (D) Example traces of mEPSCs, recorded from MSNs of wild-type adult mice in control condition and after $1 \mu \mathrm{M}$ strychnine application. (E,F) Frequency and amplitude of mEPSCs before and after strychnine application. (G) Peak-scaled non-stationary fluctuation analysis of the mEPSCs, recorded from MSNs of WT and KO animals. (H) Mean number of ultrasonic vocalizations emitted per min in male WT and KO pups at P4, P6, and P8. (I) Graph constructed from average time pups used to right on all fours. Data are presented as either box plot (whiskers indicate variability from minimum to maximum) or individual experimental values; ${ }^{*} p<0.05,{ }^{* *} p<0.01$.

of a synaptic maturation deficit in the GlyR $\alpha 2 \mathrm{KO}$, while the presynaptic vesicle release probability is unaffected by the Glra2 knockout. This decrease in the AMPA/NMDA ratio may be a consequence of a reduction of AMPARs, as shown by nonstationary fluctuation analysis of mEPSCs, rather than an increase in NMDARs.

\section{DISCUSSION}

This work establishes a link for the first time between the GlyR $\alpha 2$ subunit and the development of glutamatergic synapses in MSNs of the dorsal striatum. In the mammalian nervous system, GlyR $\alpha 2$ is mainly present during early development, with expression attenuated or disappearing in adulthood (Young-Pearse et al., 2006). By examining the expression pattern of the different GlyR subunits and glycinergic current densities during the development of the striatum, we observed a high expression of
Glra2 in neonatal striatum and corresponding glycinergic current in striatal MSNs, which decreases during maturation. In adult murine striatum, GlyR $\alpha 2$ is the main ligand binding subunit (Molchanova et al., 2018), and we confirmed here that during postnatal development, there is no elevated expression of either GlyR $\alpha 1$ or GlyR $\alpha 3$ subunits. In the MSNs of neonatal GlyR $\alpha 2 \mathrm{KO}$ mice, no glycine-evoked current was observed. Expression of Glra1 and Glra3 is still detectable at all ages examined, but significantly lower than that of Glra2. Since QPCR studies were performed in whole striatum, the interneuronal expression of Glral and 3 can contribute to detected mRNA levels (Sergeeva and Haas, 2001). We also observed a developmental shift in the $\mathrm{EC}_{50}$ and current density of evoked glycinergic responses. Considering the fact that the expression of the GlyR $\beta$ subunit gene (Glrb) does not differ between neonatal and adult MSNs, we cannot attribute this effect to receptor composition (homomeric vs. heteromeric) (Wang et al., 2006). Alternatively, this might be a consequence of either cytoplasmic modulators that affect agonist 


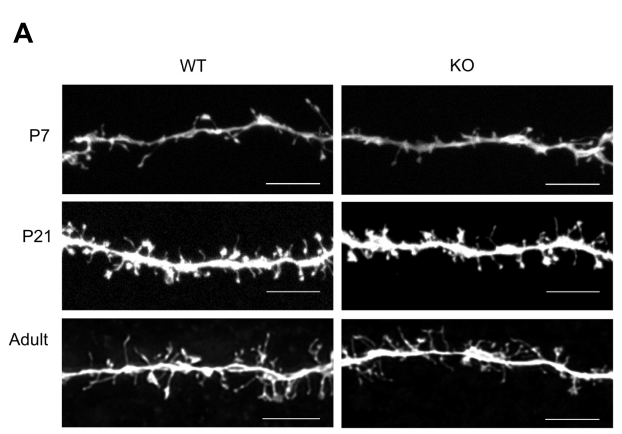

C

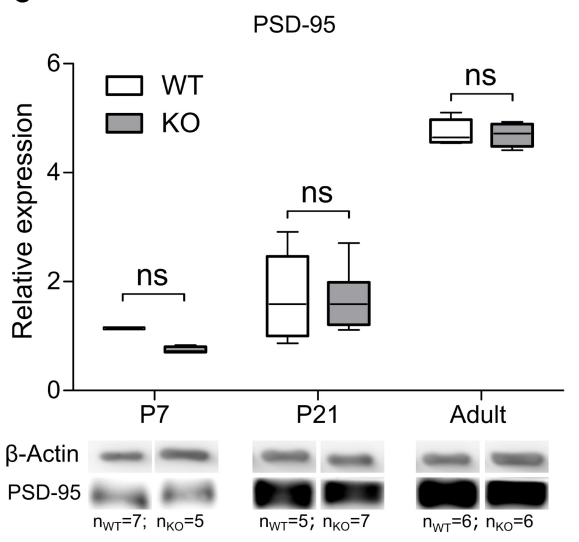

\section{B}

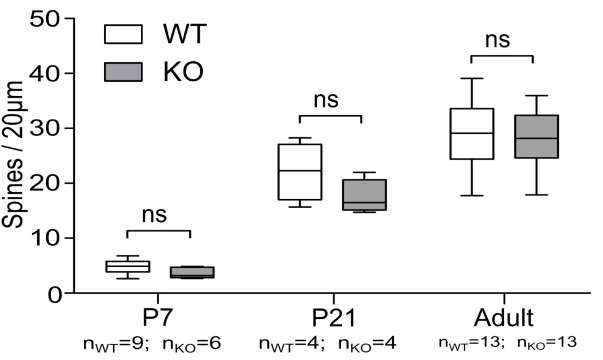

D

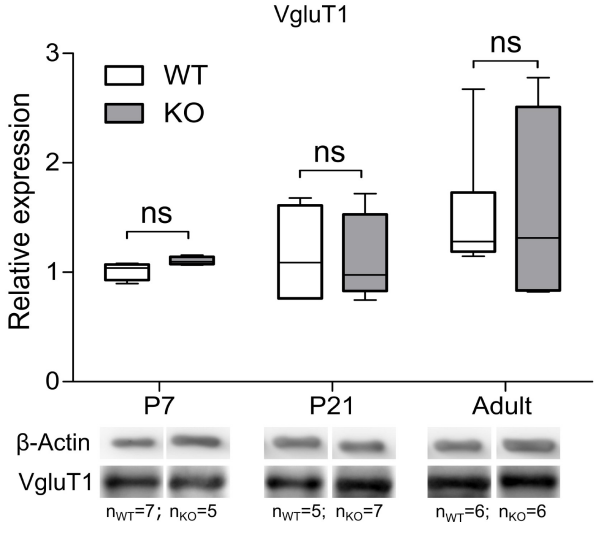

FIGURE 5 | Deletion of the GlyR $\alpha 2$ subunit does not affect the number of glutamatergic synaptic contacts on MSNs. (A) Example images of MSN spines in WT and $\mathrm{KO}$ animals of different ages. Scale bar is $5 \mu \mathrm{m}$. (B) Quantification of spine density. (C,D) Representative Western blots and quantification of the band intensity of PSD-95 (C) and VGluT1 (D) in dorsal striatum of WT and KO animals of different ages. Data are presented as box plot (whiskers indicate variability from minimum to maximum).

binding capabilities of the GlyRs or the sensitivity might also be influenced by a higher receptor density in neonates which affects GlyRs inter-receptor interactions (Taleb and Betz, 1994; Fucile et al., 2000).

Taken together, our data indicate that GlyR $\alpha 2$ is the main agonist-binding subunit of GlyRs in striatal MSNs, and that the expression of glra2 is developmentally regulated. However, the mechanism by which $\alpha 2$ subunit GlyRs mediate their actions in the neonatal MSNs seem to differ from their action in adult MSNs. In our recent study, it has been shown that GlyRs are extrasynaptic and control the RMP and excitability in adult MSNs (Molchanova et al., 2018). In neonatal MSNs, however, such tonic activity was not evident, which explains the absence of differences in RMP between GlyR $\alpha 2 \mathrm{KO}$ MSNs and wild-type controls. This is similar to the development of striatal extrasynaptic $\mathrm{GABA}_{A}$ receptors, which do not show any tonic activity up until later postnatal ages (Valtcheva et al., 2017). When blocking all inhibitory receptors, excluding the GlyR, interestingly, we did not observe any synaptic inhibitory currents, neither in neonates nor in adults (Molchanova et al., 2018). This means that functionally active GlyRs are present in neonatal MSNs, but do not seem to be activated by classic synaptic release, or extrasynaptic spill-over of neurotransmitter.
Despite the absence of intrinsic glycinergic currents, $\alpha 2$ subunit GlyRs seem to regulate the excitability of neonatal MSNs. In early developmental stages, when sensory inputs are still immature, neurons spontaneously generate the electrical activity, which serves as a testing template for newly formed synaptic connections (Blankenship and Feller, 2010). These developmental stages are called "critical periods," when neuronal networks are highly plastic and connections may change easily and form the most effective architecture to fulfill the adulttype behavioral requirements. The presence of early network activity has been shown in striatum before (Dehorter et al., 2011), and its importance regarding network formation we now also confirm. At the age of 6-7 days, roughly 30\% of striatal MSNs are spontaneously firing APs, which is in the accordance with previous findings (Dehorter et al., 2011). During development, MSNs are generated by two waves of neurogenesis, separated by 2 days in mice (Passante et al., 2008; Martin-Ibanez et al., 2017). This time gap persists during the postnatal development, and gives rise to two principal striatal compartments: patches and matrix (Brimblecombe and Cragg, 2017). The functional segregation of developing MSNs in P6-7, observed by us and others, may reflect the different time of neurogenesis and delayed development of spontaneously active cells. This is indirectly confirmed by relatively less developed RMP and evoked AP firing 
A

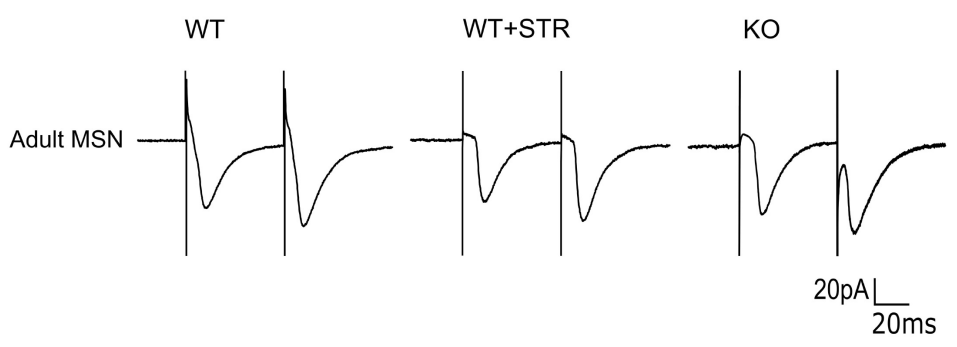

C

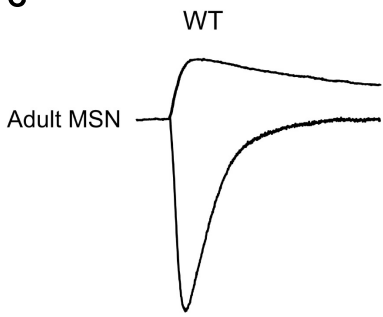

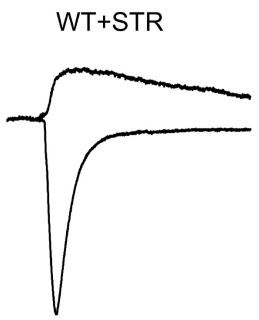

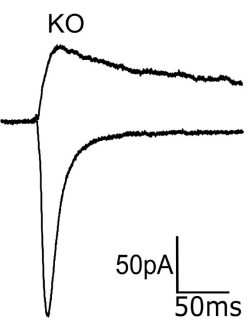

B

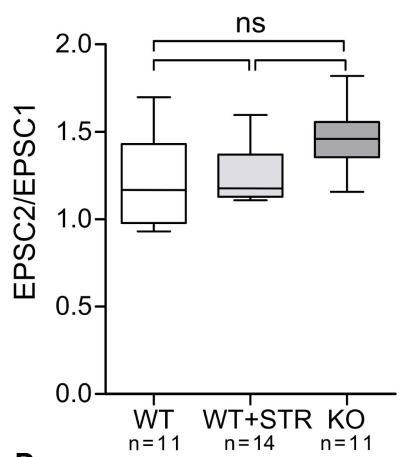

D

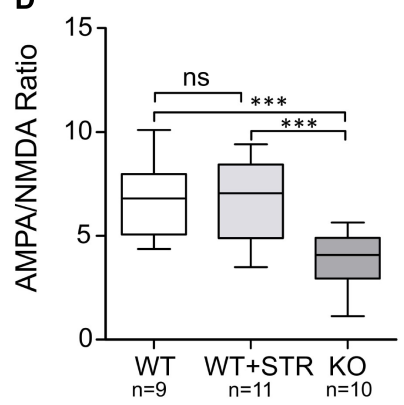

FIGURE 6 | AMPA/NMDA ratio, but not paired pulse facilitation is reduced in MSNs of adult GlyRa2KO mice. Currents were evoked by stimulation through a concentric electrode placed at the corpus callosum. (A) Example traces of paired stimulation of AMPAR-mediated currents in WT MSNs, WT MSNs in the presence of $1 \mu \mathrm{M}$ strychnine and in GlyRa2KO MSNs. Two stimuli were given with $50 \mu$ s interval. (B) Paired-pulse response ratio in control, strychnine-treated and knockout cells. (C) Example traces of AMPAR and NMDAR-mediated currents in MSNs of adult WT (control and strychnine-treated) and KO animals. AMPAR-mediated response was recorded at $-70 \mathrm{mV}$ and directed downward. NMDAR-mediated response is a result of subtraction of the response, recorded at $+40 \mathrm{mV}$ and the response in the presence of L-689,560. (D) Quantification of AMPA/NMDA ratio in MSNs of WT control, WT strychnine-treated and KO animals. Data are presented as box plot (whiskers indicate variability from minimum to maximum), ${ }^{* * *} p<0.001$.

in "active" cells, comparing to "silent" cells and MSNs of adult DS.

In GlyR $\alpha 2 \mathrm{KO}$ animals, both spontaneous and currentinduced AP firings were severely affected in spontaneously active MSNs of 6-7 days-old mice. Genetic loss of functional GlyRs or pharmacological blockade of GlyRs led to broadening of individual AP and decrease in frequency of spontaneous APs in active MSNs. The observed effect cannot be attributed to changed function of NMDARs. AP firing by "silent" MSNs, which may be at more advanced stage of development, was not affected, as well as AP shape in the adult animals (Molchanova et al., 2018). These findings suggest that GlyRs activate some yet unknown mechanism, specific for neonatal neurons, and shift to more classical extrasynaptic transmission later in development.

This neonatal mechanism of regulation of the AP shape by GlyRs is still unclear. Decrease in the amplitude and broadening of the APs are usually associated with loss of voltage-dependent potassium currents, mediated by either $\mathrm{Kv}$ or BK/SK channels (Bean, 2007; Kimm et al., 2015). Both Kv and Ca-activated potassium channels are voltage-dependent, and increase the conductance upon membrane depolarization. In our experiments, potassium current evoked by depolarising voltage steps did not differ between spontaneously active neonatal WT and GlyRa2KO MSNs, which rule out the involvement of voltage-activated potassium channels in the GlyR-mediated regulation of AP shape. However, in neonatal GlyR $\alpha 2 \mathrm{KO}$ MSNs we found the strong decrease in the potassium current, evoked by hyperpolarization. This current is mostly mediated by inward-rectifying potassium channels, for example Kir2 (classic inward rectifiers) and Kir3 (G-protein-gated potassium channels, GIRKs) (Hibino et al., 2010). Kir2 channels are abundantly expressed by adult MSNs, producing their hyperpolarized RMP, but the expression of these channels starts only at postnatal day 10 (Dehorter et al., 2011). GIRKS are absent in adult striatum, but are moderately expressed during late embryonic and early postnatal development. Interestingly enough, we did not find any difference in inward-rectifying potassium current in P14 MSNs, which match the developmental regulation of GIRK expression, and correlate with the temporary effect of GlyRs on AP firing. These facts make GIRKs a good candidates for downstream effectors of GlyR activation in developing MSNs. However, the physiological role of GIRKs is mostly linked to regulation of RMP and rheobase, and not to AP shape (Ehrengruber et al., 1997; Koyrakh et al., 2005). Together, our data suggest that $\alpha 2$ subunit GlyRs mediate regeneration of the AP firing in developing spontaneously active MSNs, with possible involvement of some inward-rectifying $\mathrm{K}^{+}$channels. Such mechanism has not been shown before for GlyRs. Since we did not detect endogenous phasic or tonic glycinergic current in MSNs at this developmental stage, it might be 
possible that GlyRs act though some non-canonical mechanism, similar to other ligand-gated ionotropic receptors which may activate $G$ protein-mediated pathways (Valbuena and Lerma, 2016). However, further studies are required to prove this concept.

Since GlyRs regulate the neonatal activity of MSNs, this might have long-term consequences on the formation and function of the striatal network in general. We confirmed that GlyR $\alpha 2 \mathrm{KO}$ mice have impaired development of glutamatergic, but not GABAergic, synapses onto striatal MSNs. A key feature of glutamatergic synaptic maturation is an increase in the AMPARmediated component of the post-synaptic current. Only after adequate rise in intracellular $\mathrm{Ca}^{2+}$, AMPARs will be inserted into the synapse (Hell, 2016). This synaptic maturation, evaluated by the AMPA-mEPSCs, is reduced at early postnatal stages of the MSNs which implies that in the GlyR $\alpha 2 \mathrm{KO}$ mice, a reduction of functional synapses is present (Bekkers and Stevens, 1995). We saw no effect of pharmacologically blocking GlyRs on these synaptic events indicating that no acute mechanism is involved in this observation. The behavioral data also seems to indicate a change in the striatal activity and connectivity already present at early postnatal stages. Spontaneous activity and glutamatergic innervation have both been found to cause changes in USV and time to right (Araujo et al., 2015; Chen et al., 2016). Here, we state that this connectivity defect is of a postsynaptic (dorsal striatum MSNs) origin rather than a presynaptic origin (cortical). Both at the functional (paired-pulse ratio) and molecular (VGluT1 protein expression) levels, we saw no change in cortical afferents. Considering the fact that we did not detect a reduction of the dendritic spines of the MSNs in all developmental stages, we evaluated the composition of the glutamatergic cortico-striatal synapses. This is further strengthened by findings in earlier work in which the connectivity defects were of intra-cortical nature. No evidence was found that pyramidal neurons projecting to extracortical structures were morphological or functionally affected in the GlyR $\alpha 2 \mathrm{KO}$ animals (Morelli et al., 2016). The decreased ratio of AMPA/NMDA in GlyR $\alpha 2 \mathrm{KO}$ mice suggests inadequate maturation at the post-synaptic level, implying a relatively higher number of silent synapses in GlyR $\alpha 2 \mathrm{KO}$ animals. This deficiency in maturation probably starts very early in development, since the frequency of NMDAR-mediated mEPSCs was not affected in neonates. Decrease in the frequency of AMPAR-mediated mEPSCs, together with the same frequency of NMDAR-mediated mEPSCs in P6-7 MSNs indirectly shows a relatively higher number of silent synapses in $\mathrm{KO}$ animals.

Our findings on the development of connectivity defects in GlyR $\alpha 2 \mathrm{KO}$ mice could in part explain the involvement of the GlyR $\alpha 2$ subunit mutations in the pathology of ASD and more specifically, the motor phenotypes present in these patients. ASD are characterized by two main symptom domains deficits in social interactions and restrictive, repetitive patterns of behavioral output (Fuccillo, 2016; Sungur et al., 2017). Dysfunction of the dorsal striatum is postulated to underlie the motor dysfunctions seen in patients with ASD (Arriaga et al., 2012; Shepherd, 2013). The striatum is a hub processing the sensory information for the realization of voluntary movements. Considering the high level of excitatory convergence (roughly
10:1) into the striatum, initial processing of action selection occurs at these synaptic connections (Yim et al., 2011). Deficits in these synaptic glutamatergic inputs into the dorsal striatum have been linked to repetitive behaviors in ASD (Yin, 2010). Our findings are in line with other works on animal models of ASD, showing that ASD phenotypes are often attributed to changes in glutamatergic cortical projections and consequently their synaptic integration into downstream brain regions such as the dorsal striatum (DS) (Peca et al., 2011; Tsai et al., 2012; Peixoto et al., 2016; Sungur et al., 2017).

Taken together, our findings suggest that GlyRs containing $\alpha 2$ subunit promote the early immature spontaneous activity of MSNs by facilitating the regeneration of membrane potential after AP firing. We also demonstrate that proper spontaneous activity is needed for correct maturation and function of the glutamatergic inputs into the dorsal striatal MSNs. When GlyR $\alpha 2$ function is lost, glutamatergic synapse function stays disturbed in adulthood, which is probably caused by impaired spontaneous activity of MSNs during early development. This highlights the importance of proper network formation in the dorsal striatum, which can be influenced from an early developmental stage onward.

\section{AUTHOR CONTRIBUTIONS}

SS, J-MR, BB, DG, JC, and SM designed the experiments. JC and SM performed the experiments. RH generated the Glra2-KO. JC, JD, and SM analyzed the data. JC and SM wrote the article. VB, $\mathrm{SB}$, and GM performed the ultrasonic vocalizations experiment and $\mathrm{CB}$ assisted with the analysis. All authors revised the article and agreed to be accountable for all aspects of the work.

\section{FUNDING}

The study was supported by Interuniversity Attraction Pole (IAP - P7/10) from Belgian Science Policy Office (BELSPO), FRS-FNRS, FMRE-Belgium and the Medical Research Council (G0500833), FWO research grant (1519516N), UHasselt general funding, King Baudouin Foundation and Rotary price "Hope in Head," Opening the Future (KU Leuven funds to CB), VIB, ERA-NET NEURON.

\section{ACKNOWLEDGMENTS}

The authors thank Frederic Bollet-Quivogne and Jean-Marie Vanderwinden for tutoring and help with microscopy; and Petra Bex, Rosette Beenaerts, Laetitia Cuvelier, and Delphine Houtteman for technical assistance. Elisabeth Piccard provided intellectual assistance.

\section{SUPPLEMENTARY MATERIAL}

The Supplementary Material for this article can be found online at: https://www.frontiersin.org/articles/10.3389/fnmol.2018. 00380/full\#supplementary-material 


\section{REFERENCES}

Akaike, N. (1996). Gramicidin perforated patch recording and intracellular chloride activity in excitable cells. Prog. Biophys. Mol. Biol. 65, 251-264. doi: 10.1016/S0079-6107(96)00013-2

Araujo, D. J., Anderson, A. G., Berto, S., Runnels, W., Harper, M., Ammanuel, S., et al. (2015). FoxP1 orchestration of ASD-relevant signaling pathways in the striatum. Genes Dev. 29, 2081-2096. doi: 10.1101/gad.267989.115

Arriaga, G., Zhou, E. P., and Jarvis, E. D. (2012). Of mice, birds, and men: the mouse ultrasonic song system has some features similar to humans and song-learning birds. PloS One 7:e46610. doi: 10.1371/journal.pone.0046610

Avila, A., Nguyen, L., and Rigo, J. M. (2013a). Glycine receptors and brain development. Front. Cell Neurosci. 7:184. doi: 10.3389/fncel.2013.00184

Avila, A., Vidal, P. M., Dear, T. N., Harvey, R. J., Rigo, J. M., and Nguyen, L. (2013b). Glycine receptor alpha2 subunit activation promotes cortical interneuron migration. Cell Rep. 4, 738-750. doi: 10.1016/j.celrep.2013.07.016

Avila, A., Vidal, P. M., Tielens, S., Morelli, G., Laguesse, S., Harvey, R. J., et al. (2014). Glycine receptors control the generation of projection neurons in the developing cerebral cortex. Cell Death Differ. 21, 1696-1708. doi: 10.1038/cdd. 2014.75

Bean, B. P. (2007). The action potential in mammalian central neurons. Nat. Rev. Neurosci. 8, 451-465. doi: 10.1038/nrn2148

Bekkers, J. M., and Stevens, C. F. (1995). Quantal analysis of EPSCs recorded from small numbers of synapses in hippocampal cultures. J. Neurophysiol. 73, 1145-1156. doi: 10.1152/jn.1995.73.3.1145

Ben-Ari, Y. (2002). Excitatory actions of gaba during development: the nature of the nurture. Nat. Rev. Neurosci. 3, 728-739. doi: 10.1038/nrn920

Berry, K. P., and Nedivi, E. (2017). Spine dynamics: are they all the same? Neuron 96, 43-55. doi: 10.1016/j.neuron.2017.08.008

Blankenship, A. G., and Feller, M. B. (2010). Mechanisms underlying spontaneous patterned activity in developing neural circuits. Nat. Rev. Neurosci. 11, 18-29. doi: $10.1038 / \mathrm{nrn} 2759$

Brimblecombe, K. R., and Cragg, S. J. (2017). The striosome and matrix compartments of the striatum: a path through the labyrinth from neurochemistry toward function. ACS Chem. Neurosci. 8, 235-242. doi: 10.1021/acschemneuro.6b00333

Chand, A. N., Galliano, E., Chesters, R. A., and Grubb, M. S. (2015). A distinct subtype of dopaminergic interneuron displays inverted structural plasticity at the axon initial segment. J. Neurosci. 35, 1573-1590. doi: 10.1523/JNEUROSCI. 3515-14.2015

Chen, Y. C., Kuo, H. Y., Bornschein, U., Takahashi, H., Chen, S. Y., Lu, K. M., et al. (2016). Foxp2 controls synaptic wiring of corticostriatal circuits and vocal communication by opposing Mef2c. Nat. Neurosci. 19, 1513-1522. doi: $10.1038 / \mathrm{nn} .4380$

Cherubini, E., Griguoli, M., Safiulina, V., and Lagostena, L. (2011). The depolarizing action of GABA controls early network activity in the developing hippocampus. Mol. Neurobiol. 43, 97-106. doi: 10.1007/s12035-010-8147-z

D’Angelo, E., De Filippi, G., Rossi, P., and Taglietti, V. (1995). Synaptic excitation of individual rat cerebellar granule cells in situ: evidence for the role of NMDA receptors. J. Physiol. 484, 397-413. doi: 10.1113/jphysiol.1995.sp020673

Dehorter, N., Michel, F. J., Marissal, T., Rotrou, Y., Matrot, B., Lopez, C., et al. (2011). Onset of pup locomotion coincides with loss of NR2C/D-mediated cortico-striatal EPSCs and dampening of striatal network immature activity. Front. Cell. Neurosci. 5:24. doi: 10.3389/fncel.2011.00024

Ebert, D. H., and Greenberg, M. E. (2013). Activity-dependent neuronal signalling and autism spectrum disorder. Nature 493, 327-337. doi: 10.1038/nature11860

Ehrengruber, M. U., Doupnik, C. A., Xu, Y., Garvey, J., Jasek, M. C., Lester, H. A., et al. (1997). Activation of heteromeric $G$ protein-gated inward rectifier $\mathrm{K}(+)$ channels overexpressed by adenovirus gene transfer inhibits the excitability of hippocampal?neurons. Proc. Natl. Acad. Sci. U.S.A. 94, 7070-7075. doi: 10.1073/ pnas.94.13.7070

Ey, E., Torquet, N., Le Sourd, A. M., Leblond, C. S., Boeckers, T. M., Faure, P., et al. (2013). The autism ProSAP1/shank2 mouse model displays quantitative and structural abnormalities in ultrasonic vocalisations. Behav. Brain Res. 256, 677-689. doi: 10.1016/j.bbr.2013.08.031

Fleischer, W., Theiss, S., Schnitzler, A., and Sergeeva, O. (2017). Glutamine triggers long-lasting increase in striatal network activity in vitro. Exp. Neurol. 290, 41-52. doi: 10.1016/j.expneurol.2017.01.003
Fuccillo, M. V. (2016). Striatal circuits as a common node for autism pathophysiology. Front. Neurosci. 10:27. doi: 10.3389/fnins.2016.00027

Fucile, S., De Saint Jan, D., de Carvalho, L. P., and Bregestovski, P. (2000). Fast potentiation of glycine receptor channels of intracellular calcium in neurons and transfected cells. Neuron 28, 571-583. doi: 10.1016/S0896-6273(00)00134-3

Hartveit, E., and Veruki, M. L. (2007). Studying properties of neurotransmitter receptors by non-stationary noise analysis of spontaneous postsynaptic currents and agonist-evoked responses in outside-out patches. Nat. Protoc. 2, 434-448. doi: 10.1038/nprot.2007.47

Hell, J. W. (2016). How Ca2+-permeable AMPA receptors, the kinase PKA, and the phosphatase PP2B are intertwined in synaptic LTP and LTD. Sci. Signal. 9:e2. doi: 10.1126/scisignal.aaf7067

Hibino, H., Inanobe, A., Furutani, K., Murakami, S., Findlay, I., and Kurachi, Y. (2010). Inwardly rectifying potassium channels: their structure, function, and physiological roles. Physiol. Rev. 90, 291-366. doi: 10.1152/physrev.00021.2009

Huupponen, J., Molchanova, S. M., Lauri, S. E., and Taira, T. (2013). Ongoing intrinsic synchronous activity is required for the functional maturation of CA3CA1 glutamatergic synapses. Cereb. Cortex 23, 2754-2764. doi: 10.1093/cercor/ bhs 262

Jamann, N., Jordan, M., and Engelhardt, M. (2018). Activity-dependent axonal plasticity in sensory systems. Neuroscience 368, 268-282. doi: 10.1016/j. neuroscience.2017.07.035

Jiang, Z. G., and North, R. A. (1991). Membrane properties and synaptic responses of rat striatal neurones in vitro. J. Physiol. 443, 533-553. doi: 10.1113/jphysiol. 1991.sp018850

Kerschensteiner, D. (2014). Spontaneous network activity and synaptic development. Neuroscientist 20, 272-290. doi: 10.1177/1073858413510044

Kilb, W., Ikeda, M., Uchida, K., Okabe, A., Fukuda, A., and Luhmann, H. J. (2002). Depolarizing glycine responses in cajal-retzius cells of neonatal rat cerebral cortex. Neuroscience 112, 299-307. doi: 10.1016/S0306-4522(02)00071-4

Kim, K. S., Kim, H., Park, S. K., and Han, P. L. (2012). The dorsal striatum expressing adenylyl cyclase- 5 controls behavioral sensitivity of the righting reflex to high-dose ethanol. Brain Res. 1489, 27-36. doi: 10.1016/j.brainres.2012. 10.016

Kimm, T., Khaliq, Z. M., and Bean, B. P. (2015). Differential regulation of action potential shape and burst-frequency firing by BK and Kv2 channels in substantia nigra dopaminergic neurons. J. Neurosci. 35, 16404-16417. doi: 10.1523/JNEUROSCI.5291-14.2015

Koyrakh, L., Lujan, R., Colon, J., Karschin, C., Kurachi, Y., Karschin, A., et al. (2005). Molecular and cellular diversity of neuronal G-protein-gated potassium channels. J. Neurosci. 25, 11468-11478. doi: 10.1523/JNEUROSCI.3484-05. 2005

Kozorovitskiy, Y., Saunders, A., Johnson, C. A., Lowell, B. B., and Sabatini, B. L. (2012). Recurrent network activity drives striatal synaptogenesis. Nature 485, 646-650. doi: 10.1038/nature11052

Lambot, L., Chaves Rodriguez, E., Houtteman, D., Li, Y., Schiffmann, S. N., and Gall, D. (2016). A. de Kerchove d'Exaerde, striatopallidal neuron NMDA receptors control synaptic connectivity, locomotor, and goal-directed behaviors. J. Neurosci. 36, 4976-4992. doi: 10.1523/JNEUROSCI.2717-15.2016

Lein, E. S., Belgard, T. G., Hawrylycz, M., and Molnar, Z. (2017). Transcriptomic perspectives on neocortical structure, development, evolution, and disease. Annu. Rev. Neurosci. 40, 629-652. doi: 10.1146/annurev-neuro-070815-013858

Malosio, M. L., Marquèze-Pouey, B., Kuhse, J., and Betz, H. (1991). Widespread expression of glycine receptor subunit mRNAs in the adult and developing rat brain. EMBO J. 10, 2401-2409. doi: 10.1002/j.1460-2075.1991.tb07779.x

Martin-Ibanez, R., Pardo, M., Giralt, A., Miguez, A., Guardia, I., Marion-Poll, L., et al. (2017). Helios expression coordinates the development of a subset of striatopallidal medium spiny neurons. Development 144 1566-1577. doi: 10. 1242/dev.138248

Molchanova, S. M., Comhair, J., Karadurmus, D., Piccart, E., Harvey, R. J., Rigo, J. -M., et al. (2018). Tonically active $\alpha 2$ subunit-containing glycine receptors regulate the excitability of striatal medium spiny neurons. Front. Mol. Neurosci. 10:442. doi: 10.3389/fnmol.2017.00442

Morelli, G., Avila, A., Ravanidis, S., Aourz, N., Neve, R. L., Smolders, I., et al. (2016). Cerebral cortical circuitry formation requires functional glycine receptors. Cereb. Cortex 27, 1863-1877. doi: 10.1093/cercor/bhw025

Passante, L., Gaspard, N., Degraeve, M., Frisen, J., Kullander, K., De Maertelaer, V., et al. (2008). Temporal regulation of ephrin/Eph signalling is required for the 
spatial patterning of the mammalian striatum. Development 135, 3281-3290. doi: $10.1242 /$ dev.024778

Peca, J., Feliciano, C., Ting, J. T., Wang, W., Wells, M. F., Venkatraman, T. N., et al. (2011). Shank3 mutant mice display autistic-like behaviours and striatal dysfunction. Nature 472, 437-442. doi: 10.1038/nature09965

Peixoto, R. T., Wang, W., Croney, D. M., Kozorovitskiy, Y., and Sabatini, B. L. (2016). Early hyperactivity and precocious maturation of corticostriatal circuits in shank3b(-/-) mice. Nat. Neurosci. 19, 716-724. doi: 10.1038/nn.4260

Pfaffl, M. W. (2001). A new mathematical model for relative quantification in real-time RT-PCR. Nucleic Acids Res. 29:e45. doi: 10.1093/nar/29.9.e45

Pilorge, M., Fassier, C., Le Corronc, H., Potey, A., Bai, J., De Gois, S., et al. (2016). Genetic and functional analyses demonstrate a role for abnormal glycinergic signaling in autism. Mol. Psychiatry 21, 936-945. doi: 10.1038/mp.2015.139

Piton, A., Gauthier, J., Hamdan, F. F., Lafreniere, R. G., Yang, Y., Henrion, E., et al. (2011). Systematic resequencing of X-chromosome synaptic genes in autism spectrum disorder and schizophrenia. Mol. Psychiatry 16, 867-880. doi: $10.1038 / \mathrm{mp} .2010 .54$

Scattoni, M. L., Gandhy, S. U., Ricceri, L., and Crawley, J. N. (2008). Unusual repertoire of vocalizations in the BTBR $\mathrm{T}+\mathrm{tf} / \mathrm{J}$ mouse model of autism. PLoS One 3:e3067. doi: 10.1371/journal.pone.0003067

Schindelin, J., Arganda-Carreras, I., Frise, E., Kaynig, V., Longair, M., Pietzsch, T., et al. (2012). Fiji: an open-source platform for biological-image analysis. Nat. Methods 9, 676-682. doi: 10.1038/nmeth.2019

Sergeeva, O. A., and Haas, H. L. (2001). Expression and function of glycine receptors in striatal cholinergic interneurons from rat and mouse. Neuroscience 104, 1043-1055. doi: 10.1016/S0306-4522(01)00130-0

Shepherd, G. M. G. (2013). Corticostriatal connectivity and its role in disease. Nat. Rev. Neurosci. 14, 278-291. doi: 10.1038/nrn3469

Sungur, A. O., Schwarting, R. K. W., and Wohr, M. (2017). Behavioral phenotypes and neurobiological mechanisms in the shank1 mouse model for autism spectrum disorder: a translational perspective. Behav. Brain Res. 352, 46-61. doi: 10.1016/j.bbr.2017.09.038

Surmeier, D. J., Ding, J., Day, M., Wang, Z., and Shen, W. (2007). D1 and D2 dopamine-receptor modulation of striatal glutamatergic signaling in striatal medium spiny neurons. Trends Neurosci. 30, 228-235. doi: 10.1016/j.tins.2007. 03.008

Taleb, O., and Betz, H. (1994). Expression of the human glycine receptor alpha 1 subunit in Xenopus oocytes: apparent affinities of agonists increase at high receptor density. EMBO J. 13, 1318-1324. doi: 10.1002/j.1460-2075.1994. tb06384.x
Tsai, P. T., Hull, C., Chu, Y., Greene-Colozzi, E., Sadowski, A. R., Leech, J. M., et al. (2012). Autistic-like behaviour and cerebellar dysfunction in purkinje cell Tsc1 mutant mice. Nature 488, 647-651. doi: 10.1038/nature11310

Valbuena, S., and Lerma, J. (2016). Non-canonical signaling, the hidden life of ligand-gated ion channels. Neuron 92, 316-329. doi: 10.1016/j.neuron.2016.10. 016

Valtcheva, S., Paille, V., Dembitskaya, Y., Perez, S., Gangarossa, G., Fino, E., et al. (2017). Developmental control of spike-timing-dependent plasticity by tonic gabaergic signaling in striatum. Neuropharmacology 121, 261-277. doi: 10.1016/ j.neuropharm.2017.04.012

Vogt, K. (2015). Diversity in gabaergic signaling. Adv. Pharmacol. 73, 203-222. doi: 10.1016/bs.apha.2014.11.009

Wang, D. S., Mangin, J. M., Moonen, G., Rigo, J. M., and Legendre, P. (2006). Mechanisms for picrotoxin block of alpha2 homomeric glycine receptors. J. Biol. Chem. 281 3841-3855.

Yamasaki, T., Maekawa, T., Fujita, T., and Tobimatsu, S. (2017). Connectopathy in autism spectrum disorders: a review of evidence from visual evoked potentials and diffusion magnetic resonance imaging. Front. Neurosci. 11:627. doi: 10. 3389/fnins.2017.00627

Yim, M. Y., Aertsen, A., and Kumar, A. (2011). Significance of input correlations in striatal function. PLoS Comput. Biol. 7:e1002254. doi: 10.1371/journal.pcbi. 1002254

Yin, H. H. (2010). The sensorimotor striatum is necessary for serial order learning. J. Neurosci. 30, 14719-14723. doi: 10.1523/JNEUROSCI.398910.2010

Young-Pearse, T. L., Ivic, L., Kriegstein, A. R., and Cepko, C. L. (2006). Characterization of mice with targeted deletion of glycine receptor alpha $2 . \mathrm{Mol}$. Cell. Biol. 26, 5728-5734. doi: 10.1128/MCB.00237-06

Conflict of Interest Statement: The authors declare that the research was conducted in the absence of any commercial or financial relationships that could be construed as a potential conflict of interest.

Copyright (c) 2018 Comhair, Devoght, Morelli, Harvey, Briz, Borrie, Bagni, Rigo, Schiffmann, Gall, Brône and Molchanova. This is an open-access article distributed under the terms of the Creative Commons Attribution License (CC BY). The use, distribution or reproduction in other forums is permitted, provided the original author(s) and the copyright owner(s) are credited and that the original publication in this journal is cited, in accordance with accepted academic practice. No use, distribution or reproduction is permitted which does not comply with these terms. 